\title{
Beam-beam observations in the Relativistic Heavy Ion Collider
}

\author{
Y. Luo, W. Fischer, S. White
}

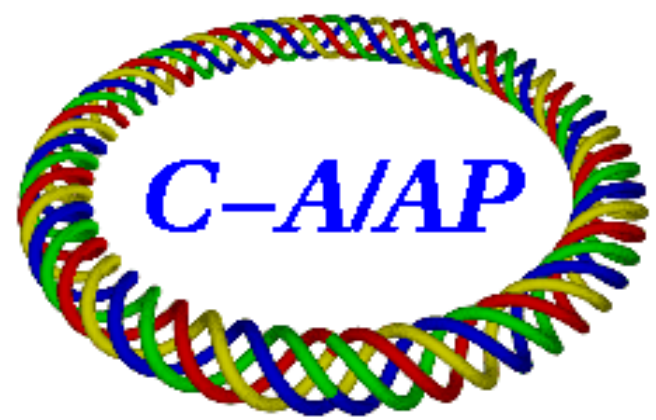

\section{Collider-Accelerator Department Brookhaven National Laboratory Upton, NY 11973}

\section{U.S. Department of Energy}

\section{Office of Science, Office of Nuclear Physics}

Notice: This document has been authorized by employees of Brookhaven Science Associates, LLC under Contract No. DE-SC0012704 with the U.S. Department of Energy. The United States Government retains a nonexclusive, paid-up, irrevocable, world-wide license to publish or reproduce the published form of this document, or allow others to do so, for United States Government purposes. 
June 24, 2015

\title{
Beam-beam observations in the Relativistic Heavy Ion Collider
}

\author{
Y. Luo, W. Fischer, and S. White \\ Brookhaven National Laboratory, Upton, NY 11973, USA
}

The Relativistic Heavy Ion Collider (RHIC) at Brookhaven National Laboratory has been operating since 2000. Over the past decade, thanks to the continuously increased bunch intensity and reduced $\beta^{*} \mathrm{~s}$ at the interaction points, the maximum peak luminosity in the polarized proton operation has been increased by more than two orders of magnitude. In this article, we first present the beam-beam observations in the previous RHIC polarized proton runs. Then we analyze the mechanisms for the beam loss and emittance growth in the presence of beam-beam interaction. The operational challenges and limitations imposed by beam-beam interaction and their remedies are also presented. In the end, we briefly introduce head-on beam-beam compensation with electron lenses in RHIC.

\section{Introduction}

The Relativistic Heavy Ion Collider (RHIC) at Brookhaven National Laboratory consists of two superconducting rings, the "Blue" ring and the "Yellow" ring. The two rings intersect horizontally at 6 symmetric Interaction Points (IPs) along a $3.8 \mathrm{~km}$ circumference. During the physics stores, the two beams collide head-on at IP6 (STAR detector) and IP8 (PHENIX detector). At other 4 non-collisional IPs, the two beams are always separated vertically. Figure 1 shows the layout of RHIC.

RHIC is capable of colliding heavy ions and polarized protons. For the gold-gold ion collision with the ion energy $100 \mathrm{GeV} /$ nucleon, the total incoherent beam-beam tune shift or the total beam-beam parameter is 0.003 with 2 collisions per turn. With stochastic cooling, the total beam-beam parameter could reach 0.01 . In the polarized proton (p-p) operation, the total beam-beam parameter is 0.014 in the $2012100 \mathrm{GeV}$ and $2013255 \mathrm{GeV}$ runs. A total beam-beam parameter larger than 0.018 was reached in the $2009100 \mathrm{GeV}$ run at the cost of beam lifetime and proton polarization. In this article, we only discuss the beam-beam effects in the RHIC p-p operation.

Over the past decade, thanks to the continuously increased bunch intensities and reduced $\beta^{*} \mathrm{~s}$ at the collisional IPs, the maximum peak luminosity in the RHIC p-p operation has increased by more than two orders of magnitude [1]. In the $2012100 \mathrm{GeV}$ p-p run, the maximum peak luminosity reached $46 \times 10^{30} \mathrm{~cm}^{-2} \mathrm{~s}^{-1}$ with a proton bunch intensity $1.65 \times 10^{11}$ and $\beta^{*}=0.7 \mathrm{~m}$. In the $2013255 \mathrm{GeV}$ p-p run, the maximum peak luminosity reached $245 \times 10^{30} \mathrm{~cm}^{-2} \mathrm{~s}^{-1}$ with a proton bunch intensity $1.85 \times 10^{11}$ and $\beta^{*}=0.65 \mathrm{~m}$.

With the increase in the proton bunch intensity, the beam-beam parameter has increased too. Beambeam interaction introduces amplitude-dependent tune spread and excites nonlinear resonance driving terms (RDTs). The current working points for the RHIC p-p operation are constrained between 2/3 and 7/10. $2 / 3$ is a strong third order betatron resonance. $7 / 10$ is a 10 th order betatron resonance and a depolarization resonance. In the $2013255 \mathrm{GeV}$ p-p run, limited by the tune space, we observed increased beam losses and emittance blow-ups when the proton bunch intensities were higher than $1.85 \times 10^{11}$. Beam-beam interaction has become the main limitation to further improve the luminosity in RHIC p-p operation.

In this article, we will first present the beam-beam observations in the previous p-p runs. Then we analyze the mechanisms for the proton particle loss and emittance growth in the presence of beam-beam interaction. The operational challenges and limitations imposed by the beam-beam interaction and their remedies are discussed. In the end we briefly introduce head-on beam-beam compensation with electron lenses in RHIC. However, the latest results with electron lenses in the $2015100 \mathrm{GeV}$ p-p run will not be included. 


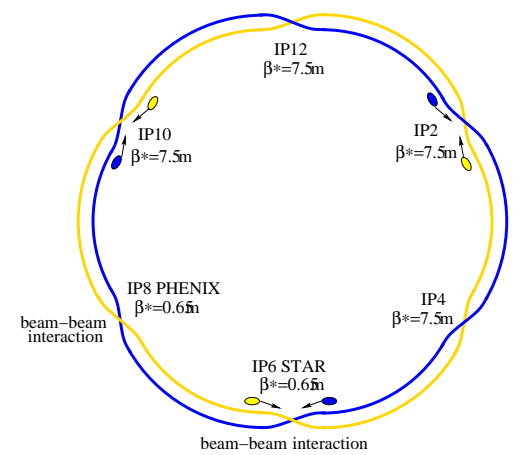

Figure 1: Layout of RHIC. Particles circulate clockwise in the BLue ring and counter-clockwise in the Yellow ring.

\section{Previous RHIC p-p Runs}

\subsection{Luminosity and Beam Parameters}

The Figure Of Merit (FOM) in the RHIC p-p operation is $L P^{2}$ for the single spin program and $L P^{4}$ for the double spin program, where $L$ is the luminosity and $P$ is the polarization of proton beams. Therefore, to maximize the FOM in the p-p operation, we should maximize both luminosity and polarization and their lifetimes at physics stores.

Polarization is the average value of the projections of proton spins on the average spin direction. The design spin direction at the collisional IPs are in the vertical direction in the single spin program and in the longitudinal direction in the double spin program. The averaged proton polarization was $60 \%$ in the 2012 $100 \mathrm{GeV}$ run and $52 \%$ in the $2013255 \mathrm{GeV}$ operation respectively [1].

The luminosity in the RHIC p-p operation is given by

$$
L=\frac{N_{p}^{2} N_{c} \gamma f_{r e v}}{4 \pi \epsilon_{n, r m s} \beta^{*}} H\left(\beta^{*}, \sigma_{l}\right),
$$

where $N_{p}$ is the proton bunch intensity, $N_{c}$ the number of collisions per turn, $\gamma$ the Lorentz factor, and $f_{\text {rev }}$ the revolution frequency. $\epsilon_{n, r m s}$ is the normalized rms transverse emittance, $\sigma_{l}$ the normalized rms bunch length. $H\left(\beta^{*}, \sigma_{l}\right)$ is the luminosity reduction factor due to the hour-glass effect. To increase luminosity, we increase the bunch intensity and reduce $\beta^{*}$ s at collisional IPs. Table 1 lists the maximum proton bunch intensities, $\beta^{*} \mathrm{~s}$, and achieved maximum peak luminosities in the previous RHIC p-p runs [1]. Table 2 lists the machine and beam parameters for the latest $2012100 \mathrm{GeV}$ and $2013255 \mathrm{GeV}$ p-p runs.

The first $100 \mathrm{GeV}$ p-p collision in RHIC took place in 2002 with a maximum peak luminosity $1.8 \times$ $10^{30} \mathrm{~cm}^{-2} \mathrm{~s}^{-1}$ with a bunch intensity $0.75 \times 10^{11}$ and $\beta^{*}=3.0 \mathrm{~m}$ at the collisional IPs [2]. In the following $100 \mathrm{GeV}$ p-p runs until $2008[3,4,5,6,7]$, the peak bunch intensity increased to $1.5 \times 10^{11}$ and $\beta^{*}$ s reduced to $1 \mathrm{~m}$. In the $2009100 \mathrm{GeV}$ run [8], the $\beta^{*}$ s were further reduced to $0.7 \mathrm{~m}$ but we observed short intensity and luminosity lifetimes. Therefore, in the following $100 \mathrm{GeV}$ run in 2012 [9], we increased $\beta^{*}$ to $0.85 \mathrm{~m}$.

Polarized protons were first accelerated to and collided at $205 \mathrm{GeV}$ in 2005 [10] and $250 \mathrm{GeV}$ in 2006. In 2009 [11], we had the first p-p physics run with the proton energy $250 \mathrm{GeV}$. The maximum peak luminosity was $20 \times 10^{30} \mathrm{~cm}^{-2} \mathrm{~s}^{-1}$ with a bunch intensity $1.1 \times 10^{11}$ and $\beta^{*}=0.7 \mathrm{~m}$. In the following $250 \mathrm{GeV}$ p-p run in 2011, and $255 \mathrm{GeV}$ p-p runs in 2012 and 2013 [12, 13, 14, 15], $\beta^{*} \mathrm{~s}$ at the collisional IPs were $0.65 \mathrm{~m}$. A maximum peak luminosity of $245 \times 10^{30} \mathrm{~cm}^{-2} \mathrm{~s}^{-1}$ was achieved in 2013 with a bunch intensity $1.85 \times 10^{11}$. The motivation for colliding protons at $255 \mathrm{GeV}$ instead of $250 \mathrm{GeV}$ is to improve the polarization lifetime at store.

The choice of working points in the RHIC p-p operation is determined by the polarization transmission efficiency on the energy acceleration and the lifetimes of intensity and polarization at store. The original design tunes for the RHIC p-p operation are $(28.18,29.19)$, which is the same as the design tunes for the gold ion operation. In the $2002100 \mathrm{GeV}$ run, we observed a poor beam lifetime with collision with these tunes. In 2004, a new working space between $2 / 3$ and $7 / 10$ was found to provide a better beam lifetime with collision as well as a better polarization transmission efficiency on acceleration. This tune space has been used up to now. 
Table 1: Maximum bunch intensities, $\beta^{*}$ s at the collisional IPs, and maximum peak luminosity in the previous RHIC p-p runs

\begin{tabular}{ccccc}
\hline \hline Runs & Energy & $\begin{array}{c}\text { Maximum } \\
\text { Bunch Intensity } \\
10^{11}\end{array}$ & $\beta^{*}$ & $\begin{array}{c}\text { Maximum } \\
\text { Peak Luminosity } \\
10^{30} \mathrm{~cm}^{-2} \mathrm{~s}^{-1}\end{array}$ \\
\hline 2002 & $\mathrm{GeV}$ & 0.7 & 3.0 & 2.0 \\
2003 & 100 & 0.7 & 1.0 & 6.0 \\
2004 & 100 & 0.7 & 1.0 & 6.0 \\
2005 & 100 & 0.9 & 1.0 & 10.0 \\
& 205 & 0.6 & 2.0 & 3.0 \\
2006 & 100 & 1.35 & 1.0 & 28.0 \\
2008 & 100 & 1.5 & 1.0 & 35.0 \\
2009 & 100 & 1.35 & 0.7 & 50.0 \\
& 250 & 1.1 & 0.7 & 85.0 \\
2011 & 250 & 1.65 & 0.65 & 145.0 \\
2012 & 100 & 1.60 & 0.85 & 46.0 \\
& 255 & 1.70 & 0.65 & 165 \\
2013 & 255 & 1.85 & 0.65 & 245 \\
\hline \hline
\end{tabular}

Table 2: Beam and optics parameters for the $2012100 \mathrm{GeV}$ and $2013255 \mathrm{GeV}$ p-p runs

\begin{tabular}{lccc}
\hline \hline & & $100 \mathrm{GeV}$ & $255 \mathrm{GeV}$ \\
\hline Parameters & Unit & in Run-12 & Run-13 \\
\hline Ring circumference & $\mathrm{m}$ & \multicolumn{2}{c}{3833.8451} \\
No. of bunches & \multicolumn{1}{c}{107} & 111 \\
Bunch intensity, initial & $10^{11}$ & 1.6 & 1.85 \\
Trans. emittance, initial & $\mu \mathrm{m}$ & 3.3 & 3.5 \\
Long. emittance, initial & $\mathrm{eV} . \mathrm{s}$ & 2.0 & 2.0 \\
Working point & - & \multicolumn{2}{c}{$(0.695,0.685)$} \\
First order chromaticities & - & \multicolumn{2}{c}{$(1,1)$} \\
$\beta^{*}$ at IP6 and IP8 & $\mathrm{m}$ & 0.85 & 0.65 \\
$\beta$ at IP10/12/2/4 & $\mathrm{m}$ & 7.5 & 7.5 \\
9 MHz RF voltage & $\mathrm{kV}$ & 20 & 20 \\
$28 \mathrm{MHz}$ RF voltage & $\mathrm{kV}$ & 300 & 300 \\
$197 \mathrm{MHz}$ RF voltage & $\mathrm{kV}$ & 200 & 100 \\
rms bunch length & $\mathrm{cm}$ & 75 & 60 \\
rms momentum spread & $10^{-4}$ & 4.2 & 1.8 \\
Beam-beam parameter/IP & - & 0.07 & 0.07 \\
Peak luminosity & $10^{30} \mathrm{~cm}^{-2} \mathrm{~s}^{-1}$ & 46 & 245 \\
Average /Peak luminosity & $\%$ & 72 & 65 \\
\hline \hline
\end{tabular}




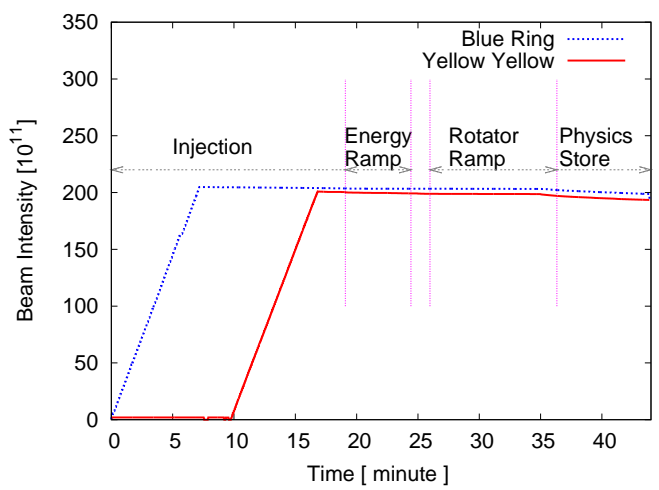

Figure 2: Operational cycle in the RHIC p-p operation. The rotator ramp is included here.

\section{$2.2 \quad$ Fill Cycle}

Figure 2 shows a typical fill cycle for the RHIC p-p operation. The horizontal axis is time in minutes. The vertical axis is the total beam intensities in the two rings. A complete cycle includes injection, energy ramp, rotator ramp, physics store, and ramp-down. The rotator ramp is designed to rotate the proton spin direction from the vertical to the longitudinal direction. For the single spin program, there is no rotator ramp.

Protons with energy $25 \mathrm{GeV}$ are injected into RHIC from its injector Alternative Gradient Synchrotron (AGS). The beams in the Blue and Yellow rings are separated vertically in the 6 common pipe interaction regions (IRs). The $\beta^{*}$ s at all IPs at injection are $10 \mathrm{~m}$. During the energy ramp, protons are accelerated to the top energy $100 \mathrm{GeV}$ or $250 / 255 \mathrm{GeV}$. The $\beta^{*}$ s at IP6 and IP8 are pre-squeezed during the energy ramp and further squeezed to their final values during the rotator ramp. At the end of the rotator ramp, the two beams are brought into head-on collision at IP 6 and IP 8 by removing the vertical separation orbit bumps there.

To maintain the beam intensity, emittance, and the polarization on the energy and rotator ramps, the orbits, tunes, betatron coupling, and chromaticities have been well controlled [16, 17, 18, 19]. Figure 3 shows the tunes during the energy ramp in the $2013255 \mathrm{GeV}$ p-p run with the tune/coupling feedback on. The tunes and global coupling are continuously measured and corrected with a base-band phase-lock-loop (PLL) tune meter. Operational experience shows that a vertical orbit with its rms value better than $0.2 \mathrm{~mm}$ and a lower vertical tune close to $2 / 3$ gives a higher polarization transmission efficiency. In Fig. 3 , the vertical tune was even temporarily pushed down to 0.671 between 190 and 220 seconds to avoid several strong depolarization resonances there.

Before 2011, only $28 \mathrm{MHz}$ RF system was used for the p-p operation. To overcome the longitudinal and transverse instabilities and to preserve the emittances at injection and on acceleration, a common $9 \mathrm{MHz}$ $\mathrm{RF}$ system has been commissioned in $2011[12,13]$. $9 \mathrm{MHz} \mathrm{RF}$ cavity provides a large bucket area and reduces the peak bunch current. Therefore, the detrimental effects from the electron cloud or impedances has been mitigated [20, 21, 22, 23]. After reaching the top energy, the bunches are re-bucketed from $9 \mathrm{MHz}$ to $28 \mathrm{MHz}$ RF system between the energy and rotator ramps. At physics stores, to produce more collisions in the central area of detectors, $300 \mathrm{KV} 197 \mathrm{MHz}$ RF voltage was added to create an even shorter bunch length. 


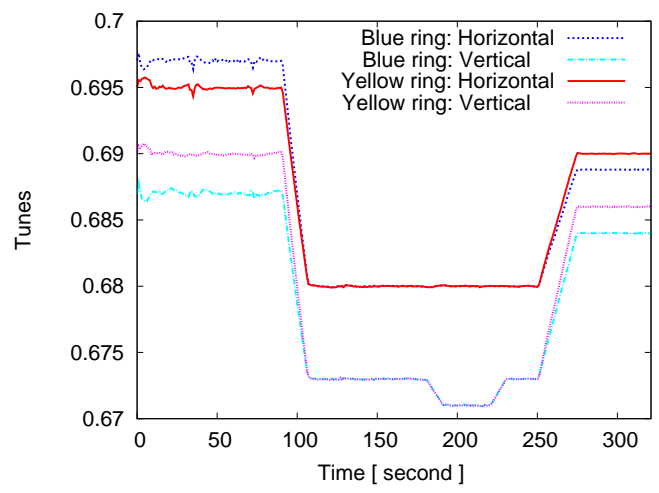

Figure 3: Tunes on the energy ramp in $2013255 \mathrm{GeV}$ p-p run. Vertical tune was pushed even closer to 2/3 between 190 to 220 seconds to achieve a higher polarization transmission efficiency.

\section{Beam-beam interaction in RHIC}

\subsection{Long-range and Head-on Beam-beam}

The RF harmonic number is 120 at injection with the common $9 \mathrm{MHz}$ RF cavity. We normally inject 109 to 111 bunches into each ring. The longitudinal distance between two adjacent bunches is $106 \mathrm{~ns}$. An empty filling gap of $1 \mathrm{~ms}$ is reserved for beam abort purpose. The bunches from different rings are separated vertically at all IPs by $20 \mathrm{~mm}$ at injection, which is 15 times the transverse rms beam sizes at IPs.

At store, the bunches are separated vertically at all non-collisional IPs by $6 \mathrm{~mm}$, which is 75 times the transverse rms beam sizes at IPs. There is one long-range beam-beam interaction in the horizontal plane on either side of collisional IP6 and IP8 before the two beams are split into their own beam pipes. The horizontal separation is $45 \mathrm{~mm}$, or 24 times the local transverse rms beam size. The impact of long-range beam-beam interaction in the RHIC p-p operation is negligible [24, 25].

There are 107 collisions at IP8 and 102 collisions at IP6 per revolution period. Most bunches have 2 collisions per turn except 10 bunches from each ring have only 1 collision per turn. For bunches with 2 collisions per turn, due to the six-folded symmetry of ring geometry and collisions only at IP6 and IP8, a group of 3 bunches from one ring will only collide with a group of 3 bunches from the other ring.

\subsection{Beam-beam Tune Shift}

For head-on beam-beam interaction, the total transverse kicks received by a test proton after one passage through the opposite proton bunch are given by [26]

$$
\left(\begin{array}{c}
\Delta x^{\prime} \\
\Delta y^{\prime}
\end{array}\right)=\frac{2 N_{p} r_{p}}{\gamma r^{2}}\left(1-e^{-\frac{r^{2}}{2 \sigma_{p}^{2}}}\right)\left(\begin{array}{l}
x \\
y
\end{array}\right)
$$

Here $r_{p}$ is the classic proton radius and $\sigma_{p}$ is the rms transverse bunch size at the interaction point. We assumed that the proton bunch has a round Gaussian transverse distribution, which is a good approximation for the RHIC p-p operation.

To calculate the linear tune shift due to the beam-beam interaction, we assume that $r / \sigma_{p} \ll 1$. Eqs. (3) is then simplified to

$$
\left(\begin{array}{c}
\Delta x^{\prime} \\
\Delta y^{\prime}
\end{array}\right)=\frac{N_{p} r_{0}}{\gamma \sigma_{p, I P}^{2}}\left(\begin{array}{l}
x \\
y
\end{array}\right)
$$

The linear beam-beam tune shift, or the beam-beam parameter per collision is

$$
\Delta Q_{x, y}=-\frac{N_{p} r_{0} \beta_{I P}^{*}}{4 \pi \gamma \sigma_{p, I P}^{2}}=-\frac{N_{p} r_{0}}{4 \pi \epsilon_{r m s}} .
$$

Here $\beta_{I P}^{*}$ is the $\beta$-function at the beam-beam interaction point. For a bunch intensity of $2.0 \times 10^{11}$ and a normalized rms transverse emittance of $2.5 \mathrm{~mm}$ mrad, the beam-beam parameter is 0.01 per IP. 


\subsection{Beam-beam Tune Spread and Resonance Driving Terms}

Beam-beam interaction also introduces amplitude-dependent tune shift and drives beam-beam resonances. The perturbed Hamiltonian including beam-beam interactions is $[28,27]$

$$
H\left(J_{x}, J_{y}, \Phi_{x}, \Phi_{y}\right)=2 \pi Q_{x} J_{x}+2 \pi Q_{y} J_{y}+\sum_{i=1}^{N_{c}} V_{i}\left(x_{n}, y_{n}\right)
$$

where $J_{x, y}$ and $\Phi_{x, y}$ are the horizontal and vertical actions and angles. $V_{i}(x, y)$ is the $i$-th beam-beam interaction potential. $x_{n}$ and $y_{n}$ are the normalized coordinates, $x_{n}=\sqrt{2 J_{x} \beta_{x i}} \cos \left(\Phi_{x}+\phi_{x i}\right)$, and $y_{n}=$ $\sqrt{2 J_{y} \beta_{y i}} \cos \left(\Phi_{y}+\phi_{y i}\right)$, where $\beta_{x i, y i}$ and $\phi_{x i, y i}$ are the betatron functions and phase advances at the $i$-th beam-beam interaction point. $N_{c}$ is the number of collisions per turn. In Eq. (5), the non-linear magnetic fields are not included.

For simplicity, we consider 1-D beam-beam interaction to calculate the beam-beam RDTs. Taking the horizontal plane as an example, the Hamiltonian is simplified as

$$
H\left(J_{x}, \Phi_{x}\right)=2 \pi Q_{x} J_{x}+\sum_{i}^{N} V_{i}\left(x_{n}\right) .
$$

with

$$
\begin{gathered}
V_{i}\left(x_{n}\right)=-\int_{0}^{x} \Delta x^{\prime} d x \\
\left.\Delta x^{\prime}\right|_{p-p}=\frac{2 N_{p} r_{0}}{\gamma} \frac{1}{x}\left(1-e^{-x^{2} / 2 \sigma_{p, I P}^{2}}\right) .
\end{gathered}
$$

Plugging Eqs.(7) and (8) into Eq. (6) and after some algebra, the beam-beam interaction potential is written as

$$
\begin{aligned}
V_{i}\left(J_{x}, \Phi_{x}\right)= & -\frac{N_{p} r_{0}}{\gamma} \int_{0}^{J_{x} \beta_{I P}^{*} / 2 \sigma_{p, I P}^{2}} \\
& \frac{d \alpha}{\alpha}\left(1-e^{-2 \alpha \cos ^{2}\left(\Phi_{x}+\phi_{x, p-p}\right)}\right) .
\end{aligned}
$$

To determine the beam-beam RDTs, we expand the beam-beam potentials into Fourier series,

$$
\sum_{i}^{N} V_{i}\left(x_{n}\right)=h_{0}\left(J_{x}\right)+\sum_{n \neq 0} h_{n}\left(J_{x}\right) e^{-i n \Phi_{x}},
$$

and the Hamiltonian is re-written as

$$
H\left(J_{x}, \Phi_{x}\right)=2 \pi Q_{x} J_{x}+h_{0}\left(J_{x}\right)+\sum_{n \neq 0} h_{n}\left(J_{x}\right) e^{-i n \Phi_{x}} .
$$

The amplitude-dependent beam-beam tune shift is then given by

$$
\Delta Q_{x}\left(J_{x}\right)=\frac{1}{2 \pi} \frac{\partial h_{0}\left(J_{x}\right)}{\partial J_{x}} .
$$

When $J_{x}=0, \Delta Q_{x}(0)$ gives the linear incoherent beam-beam tune shift as found in Eq. (4).

Figure 4 shows the beam-beam tune footprint from numeric particle tracking for the RHIC p-p operation. The different colors illustrate particles with different initial transverse amplitudes. In this example, the fractional tunes without beam-beam interaction are $(0.695,0.685)$ and $(0.685,0.695)$. The two beams collide at IP6 and IP8. The proton bunch intensity is $2.0 \times 10^{11}$ and the normalized rms transverse emittance is $2.5 \mu \mathrm{m}$. From Fig. 4, when the proton bunch intensity is larger than $2.0 \times 10^{11}$, or the total beam-beam parameter is larger than 0.02 , the beam-beam tune spread will overlap either the $2 / 3$ or $7 / 10$ resonance. There is not enough tune space between $2 / 3$ and $7 / 10$ to accommodate the beam-beam tune spread when the proton bunch intensity is larger than $2.0 \times 10^{11}$.

$h_{n}\left(J_{x}\right)$, where $n \neq 0$, is the beam-beam resonance driving term. $h_{n}\left(J_{x}\right)$ will drive the resonance $n Q_{x}=p$, where $p$ is an integer. For a round Gaussian beam, only even order beam-beam resonances are excited. Following Ref. [29, ?], we define the width of the beam-beam resonance as $d h_{n}\left(J_{x}\right) / d J_{x}$. According to Refs. [29, 30, 31, 32, 33], resonance overlapping could cause chaotic particle motion and degrade the beam lifetime. 


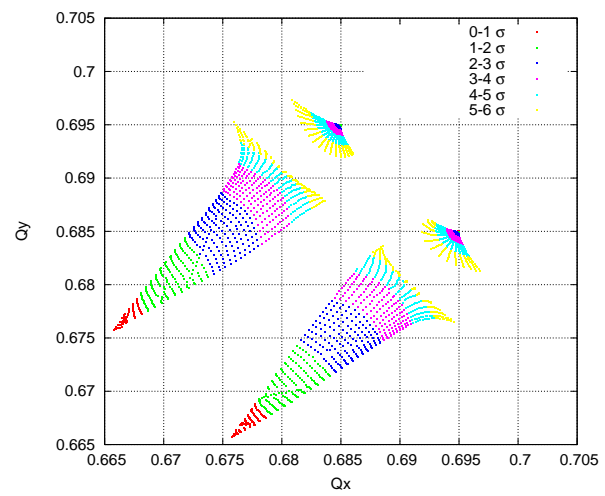

Figure 4: Tune footprints without and with beam-beam interaction with a bunch intensity $2.0 \times 10^{11}$. Different colors represent particles with different transverse amplitudes.

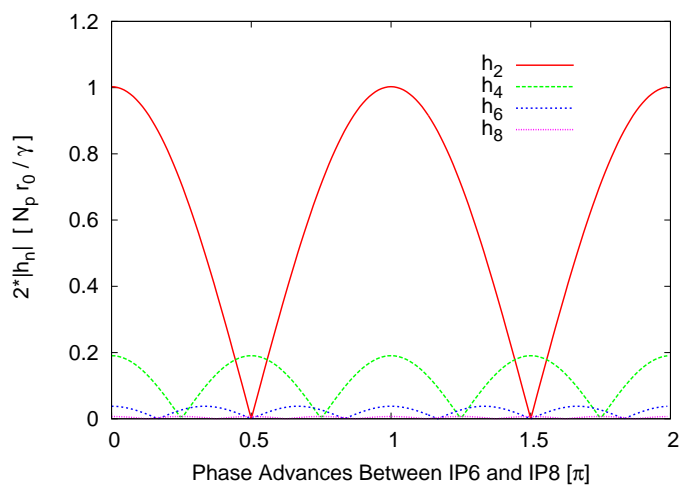

Figure 5: Beam-beam resonance driving terms as a function of the phase advance between the two collisional IPs.

As an example, Figure 5 shows the numerically calculated beam-beam RDTs as a function of the phase advance between the two collisional IPs. In this calculation, the total beam-beam parameter is 0.014 . To reduce the amplitude of the resonance $n Q_{x}$, the phase advances between IP 6 and IP 8 should be $(2 k+1 / n) \pi$, where $k$ is an integer. For an example, to reduce the half-integer resonance RDT and the dynamic $\beta$-beat due to beam-beam interaction, the phase advances between IP 6 and IP 8 should be $(2 k+1 / 2) \pi$. This phase advance specification is not implemented in the RHIC p-p operation.

\section{BEAM-BEAM OBSERVATIONS}

In this section we will present the general beam-beam observations in the previous RHIC p-p runs. We will focus on the evolutions of beam intensity, transverse emittance, bunch length, and luminosity at the routine physics stores. Since the general beam-beam observations in the $100 \mathrm{GeV}$ and 250/255 GeV p-p operations are very similar, examples given in the following are selected from the $250 / 255 \mathrm{GeV}$ runs.

\subsection{Beam Loss}

The beam intensity can be measured with DC Current Transformers (DCCTs) and Wall Current Monitors (WCMs) in RHIC. DCCTs measure both bunched and de-bunched beams, while WCMs are designed to measure only the bunched beam. WCMs are also capable of measuring the bunch intensities.

The particle loss rate $\tau^{-1}(t)$ is used in the RHIC operation system, which is defined as

$$
\tau^{-1}(t)=-\frac{1}{N_{p}(t)} \frac{d N_{p}(t)}{d t},
$$

$N_{p}(t)$ is the instant beam intensity. The unit of particle loss rate is given in $100 \% /$ hour. 

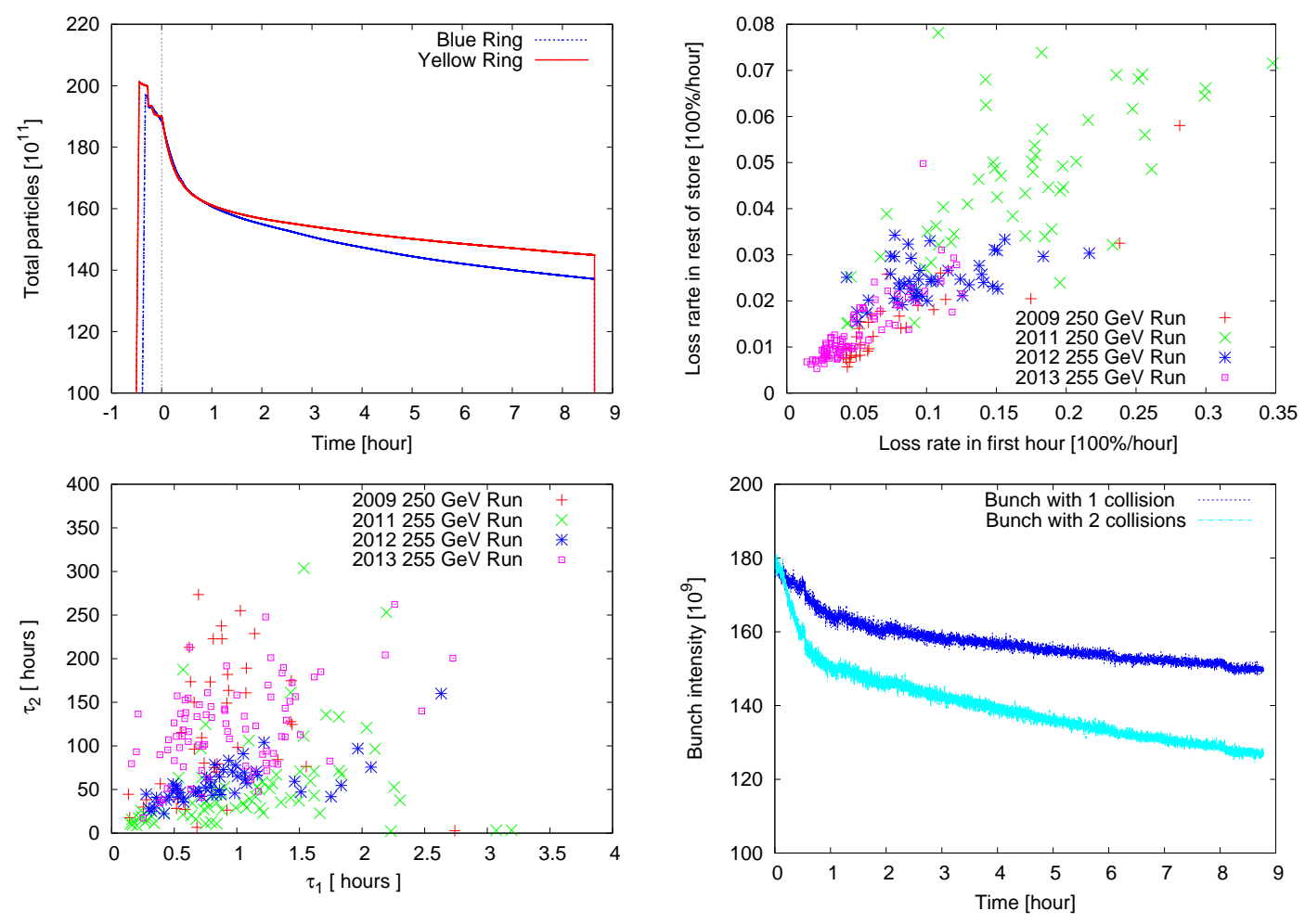

Figure 6: Top-left: Evolutions of beam intensities at store for Fill 16697 in the $2012255 \mathrm{GeV}$ run. Top-right: Averaged particle loss rates in the first hour and in the rest of store for all the physics stores in the past 250/255 GeV runs. Bottom-left: Fitting parameters $\tau_{1,2}$ in Eq. (15) for all the physics stores in the past 250/255 GeV runs. Bottom-right: Evolutions of bunch intensities of 2 bunches at store for Fill 16697: one with 1 collision and the other one with 2 collisions per turn.

Without collision at the store energies, the particle loss rate could be less than $1 \%$ /hour depending on the machine tuning. Right after we brought the two beams into collision, the particle loss rate suddenly jumped up in the first few minutes. Then the particle loss rate slowly came down in the following 1-2 hours. After that, the particle loss rate would stay below $5 \%$ /hour.

For an example, the top-left plot of Fig. 6 shows the evolutions of total beam intensities for Fill 16697 in the $2012255 \mathrm{GeV}$ run. The maximum instant particle loss rates right after collision were $35 \% /$ hour in the Blue ring and 50\%/hour in the Yellow ring. $15 \%$ of beam intensities were lost in the first hour with collision.

The top-right plot of Fig. 6 shows the average particle loss rates for all the physics stores in the past $250 / 255 \mathrm{GeV}$ runs. The horizontal axis is the average particle loss rate in the first hour. The vertical axis is the average particle loss rate in the rest of store. From this plot, the particle loss rate in the first hour was 5 times that in the rest of store. The beam loss rates in 2011 were much higher than other runs due to commissioning of the $9 \mathrm{MHz}$ RF cavity and other systems.

The evolution of beam intensity at store can be modeled with different functions. If we assume the beam loss as a diffusion process, with a constant diffusion coefficient $D$, the beam loss can be fitted with the following equation [34],

$$
N_{p}(t)=N_{p, 0}\left(1-\sqrt{t / \tau_{d}}\right),
$$

where $N_{p, 0}$ is the initial beam intensity, $\tau_{d}$ is the diffusion time. $\tau_{d}=\frac{\pi I_{0}}{4 D}, I_{0}$ is the action at the aperture boundary, $D$ is the diffusion coefficient. Since the particle loss rates are very different in the first hour and in the rest of store, we have to fit the beam intensity evolution separately in two parts with different $\tau_{d} \mathrm{~s}$.

Mathematically, the beam intensity throughout the whole store can be fitted with one expression involving two exponentials,

$$
N_{p}(t)=A_{1} \exp \left(-t / \tau_{1}\right)+A_{2} \exp \left(-t / \tau_{2}\right) .
$$

Here $A_{1,2}$ and $\tau_{1,2}$ are the fitting parameters. $\tau_{1}$ and $A_{1}$ represent mostly the early loss right after collision. The bottom-left plot of Fig. 6 shows the fitted $\tau_{1,2}$ for all the physics stores in the past $250 / 255 \mathrm{GeV}$ p-p runs. The typical $\tau_{1}$ s are between 0.5 to 1.5 hours. 

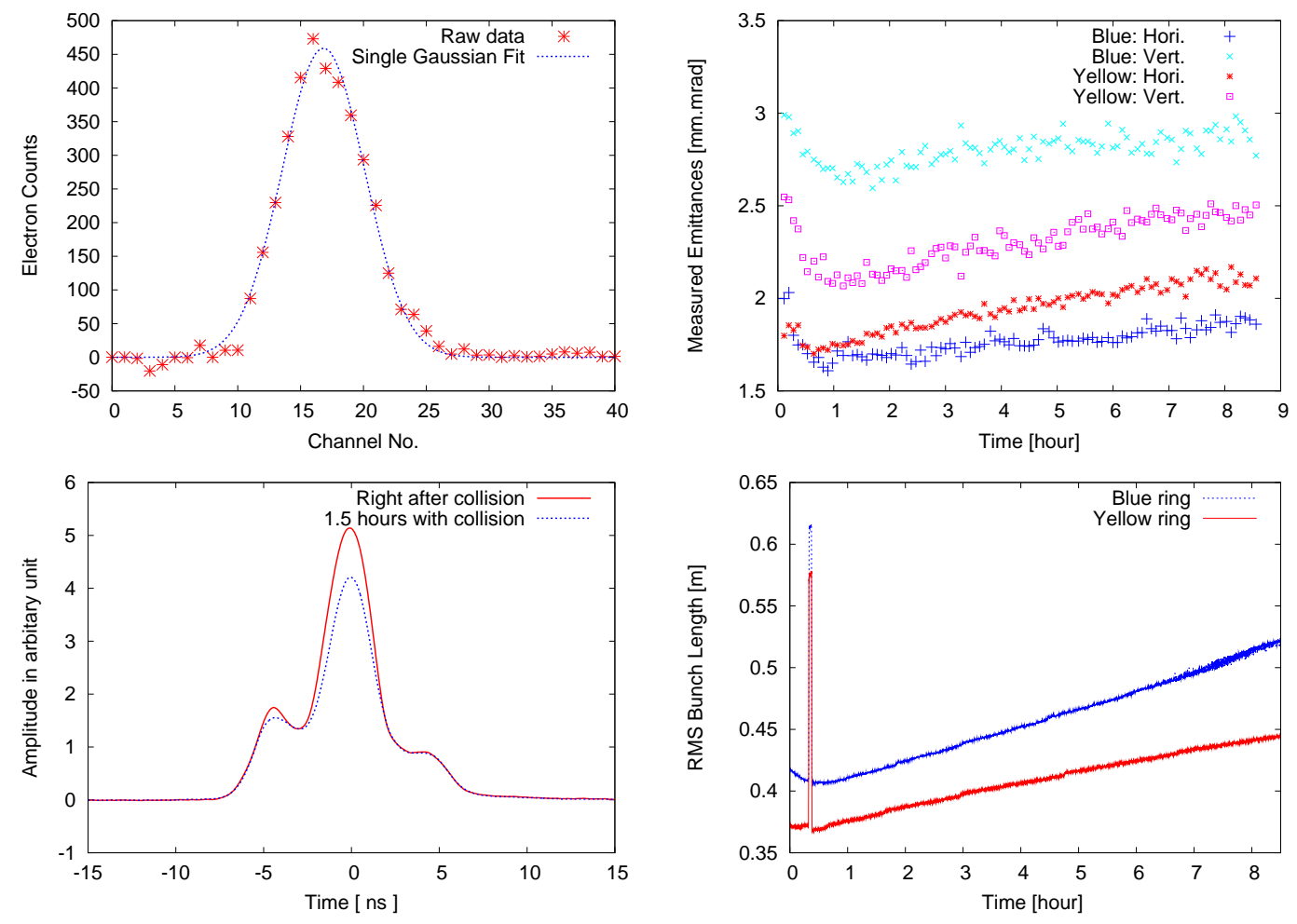

Figure 7: Top-left: an example of transverse profile measured with IPM. Top-right: Evolutions of transverse emittances at store for Fill 16697. Bottom-left: examples of longitudinal bunch profiles from WCMs, one profile taken right after collision, the other one taken 1.5 hours into store. Bottom-right: Evolutions of rms bunch lengthes for Fill 16697.

As mentioned above, there are a few bunches only having 1 collision, instead of 2 collisions per turn. The bottom-right plot of Fig. 6 shows the intensity evolutions of two adjacent bunches selected from Fill 16697: one bunch with 1 collision and the other one with 2 collisions per turn. These two bunches had very similar bunch intensities before collision. With collision, the particle loss rate for the bunch with 2 collisions was more than double that for the bunch with 1 collision.

Here we estimate the proton particle loss rate due to luminosity burn-off, which is given by

$$
\tau_{\text {burn-off }}^{-1}=\frac{N_{I P} L \sigma_{t o t}}{N_{p} N_{B}} .
$$

$N_{p}$ is the average bunch intensity, $N_{I P}$ the number of collisions per turn, and $N_{B}$ the number of bunches per ring. $\sigma_{\text {tot }}$ is the total cross section of p-p collision, which is $50 \mathrm{Mbarn}$ for the $250 \mathrm{GeV}$ p-p collision. Taking the record luminosity $L=2.38 \times 10^{32} \mathrm{~cm}^{-2} \mathrm{~s}^{-1}$ in the $250 / 255 \mathrm{GeV}$ p-p runs, together with $N_{p}=1.85 \times 10^{11}$, $N_{I P}=2$, and $N_{B}=109$, we have

$$
\tau_{\text {burn-off }}^{-1}=0.43 \% / \text { hour } .
$$

Comparing to the observed beam loss rates, the burn-off caused particle loss rate is negligible.

Considering the large beam loss with collision and the difference in beam loss rates between bunches with 1 and 2 collisions per turn, we conclude that the beam loss in the RHIC p-p run was dominated by the beam-beam interaction.

\subsection{Transverse Emittance and Bunch Length}

The transverse emittances are measured with Ionization Profile Monitors (IPMs) in RHIC. Electrons are generated when the passing protons ionize the residual gas. These electrons are collected by the IPM. The counts of electrons in different channels of IPM are associated with the transverse bunch profile. There are totally 64 channels for each IPM. The distance between adjacent channels is $0.44 \mathrm{~mm}$.

As an example, the top-left plot of Fig. 7 shows an example of transverse bunch profile measured with IPM. The horizontal axis is the IPM channel number. The red dots are the raw counts of electrons. By 
fitting the counts with a Gaussian distribution as shown by the dashed blue line, we obtain the rms transverse beam size. Together with the local $\beta$ functions at the IPM, the transverse emittance is determined.

The top-right plot of Fig. 7 shows the IPM measured emittances throughout the store of Fill 16697. The horizontal and vertical emittances of both rings are shown. In the 2011 and 2012 250/255 GeV p-p runs, the transverse emittances normally first decreased in the first hour right after collision, then slowly increased in the rest of store. We associated the emittance reduction at the beginning of store to the fast large beam loss in the first hour. Fast loss of particles with large transverse amplitudes reduces the transverse rms beam sizes.

WCMs are used to measure each bunch's longitudinal profile. The bottom-left plot of Fig. 7 shows an example of averaged longitudinal profiles of all bunches in the Blue ring for Fill 16697. The horizontal axis is the distance to the bunch center in units of ns. The vertical axis is in arbitrary unit which is proportional to the line current. Two profiles are shown in this plot: one is taken right after collision, another is taken at 1.5 hours into store.

As mentioned above, a $300 \mathrm{KV}$ of $197 \mathrm{MHz} \mathrm{RF}$ cavity was used at physics stores to obtain a shorter bunch length. The width of $197 \mathrm{MHz}$ RF bucket is $5 \mathrm{~ns}$. From Fig. 7, the longitudinal bunch profile was not Gaussian anymore. Two shoulders appeared on both sides of the central $197 \mathrm{MHz}$ RF bucket. This is due to the fact that the longitudinal emittance was too big to be fit into the central bunch.

In the RHIC operation system, Full Width Half Maximum (FWHM) bunch length is often used. For a perfect Gaussian, FWHM bunch length is 2.35 times the rms bunch length. The bottom-right plot in Fig. 7 shows an example of bunch length evolution from Fill 16697. The vertical axis is the rms bunch length derived from FWHM bunch length with a Gaussian distribution assumption. Therefore the shown rms bunch length is only meaningful for the central bunch.

From the bottom-right plot of Fig. 7, the bunch length decreased in the first hour right after collision and then slowly increased in the rest of store. We associate the early bunch length reduction to the early fast large beam loss too. Fast loss of particles in the bunch tail reduces the rms beam length. The spikes around 0.4 hour in the plot are related to polarization measurements when we reduced the voltages of $197 \mathrm{MHz} \mathrm{RF}$ voltages to $70 \mathrm{KV}$ to measure the polarization. High line current of bunches would cause sparkings around the thin wires of polarimeters.

In the $2013255 \mathrm{GeV}$ p-p run, with the same lattice used in 2011 and 2012, we reduced the voltage of $197 \mathrm{MHz}$ cavities to $100 \mathrm{KV}$ at store to obtain a better beam lifetime. Another improvement in 2013 is that longitudinal bunch-by-bunch dampers became operational and were used at injection and on the energy ramp to overcome the longitudinal instability. In the first hour, the bunch length either did not increase or increased much slower than the rest of store. The transverse emittances normally blew up right after collision and slowly grew in the rest store.

To summarize, we normally observed reductions in the transverse emittances and bunch lengths right after collision in the 2011 and 2012 p-p runs. In 2013 run, we observed emittance blow-ups and slow bunch length growths right after collision. All these phenomena are related to beam-beam interaction and will be explained in the next section.

\subsection{Store Analysis}

Emittance measurement with IPMs requires a good knowledge of the $\beta$ functions at the measurement location and a good calibration of the total 64 micro-channels. To compare with the IPM emittance measurements and for further store analysis, according to Eq. (1), we define a luminosity-derived averaged transverse emittance,

$$
<\epsilon>=\frac{N_{p}^{2} N_{c} \gamma f_{\text {rev }}}{4 \pi \beta^{*} L} H\left(\beta^{*}, \sigma_{l}\right) .
$$

Knowing instant luminosity, bunch intensity, and hour-glass factor $H$, together with known $\beta^{*}$ at collisional IPs, the luminosity-derived transverse emittance can be calculated throughout the store. However, the luminosity-derived emittance ignores the difference between the horizontal and vertical planes and the difference between the two rings.

The hour-glass luminosity reduction factor $H\left(\beta^{*}, \sigma_{1}\right)$ can be calculated precisely with the actual longitudinal bunch profiles measured with WCMs. For an example, we slice the averaged longitudinal bunch profile in each ring into $K$ slices, then

$$
H\left(\beta^{*}, \sigma_{1}\right)=\sum_{j=1}^{K} \sum_{i=1}^{K} \frac{n_{1, i} n_{2, j} \sigma^{2}(0)}{N_{p, 1} N_{p, 2} \sigma^{2}(z)},
$$




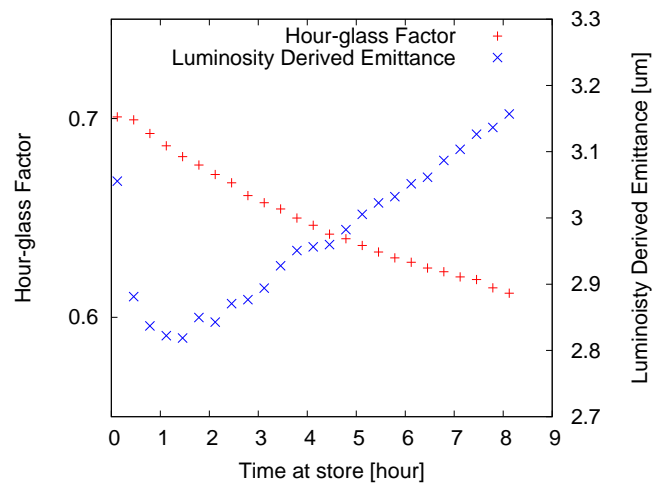

Figure 8: Hour-glass luminosity reduction factor and the luminosity-derived rms transverse emittance for Fill 16697.
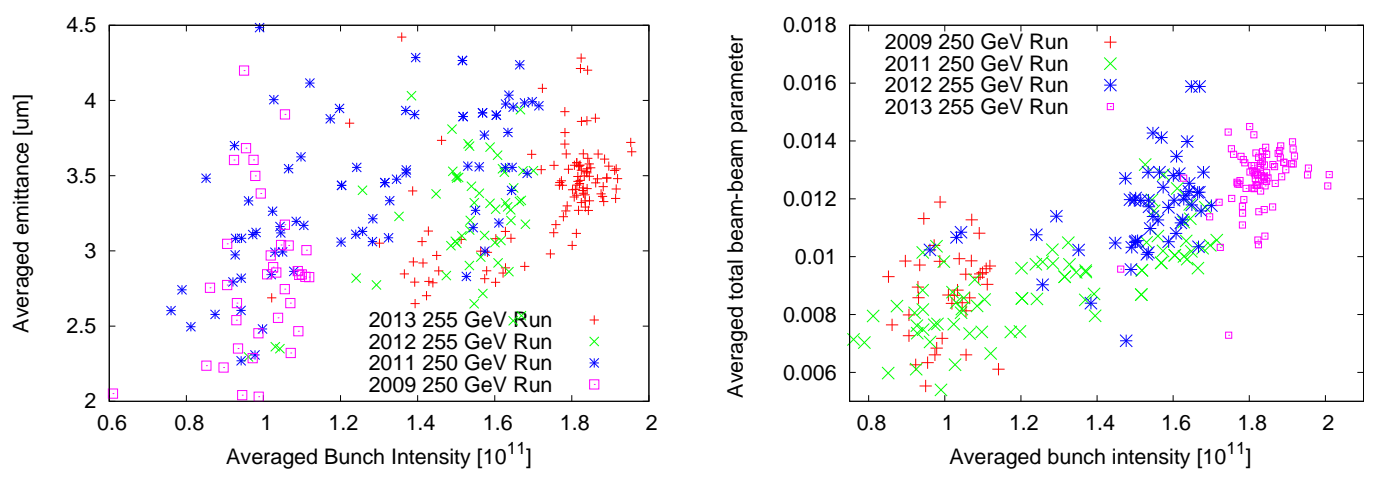

Figure 9: Left: Luminosity-deverived emittance versus the bunch intensity at the beginning fo store for all the physics fills in the previous 250/255 GeV RHIC p-p runs. Right: the total Beam-beam parameter with 2 collisions versus the averaged bunch intensities for all the physics stores in the previous $250 / 255 \mathrm{GeV}$ RHIC p-p runs.

with

$$
\frac{\sigma^{2}(0)}{\sigma^{2}(z)}=\frac{\beta^{*}(0)}{\beta^{*}(0)+\frac{z^{2}}{\beta^{*}(0)}} .
$$

$n_{1, i}$ and $n_{2, j}$ are the particle populations of the $i$-th slice of beam 1 and of the $j$-th slice of beam 2. $N_{p, 1}$ and $N_{p, 2}$ are the averaged bunch intensities of the two beams. $z$ is the longitudinal location where these two slices collide, $z=\left(z_{1, i}-z_{2, j}\right) / 2, z_{1, i}$ and $z_{2, j}$ are the relative longitudinal positions of the slices w.r.t their bunch centers.

Figure 8 show the calculated hour-glass luminosity reduction factor and the luminosity-derived averaged emittance for Fill 16697. The hour-glass factor was 0.7 at the beginning of store and decreased to 0.6 after 8 hours. The luminosity-derived emittance decreased in the first hour and then slowly increased in the rest of store, which is qualitatively in agreement with the IPM measurements. With recent improvements in IPM channel calibration and $\beta$-function measurement, the discrepancy in emittance between the IPM measurements and that derived from luminosity can be reduced to below $10 \%$.

The left plot of Fig. 9 shows the luminosity-derived emittance versus the averaged bunch intensity at the beginning of store for all physics stores in the past $250 / 255 \mathrm{GeV}$ runs. We can see that the emittances slightly increased with the bunch intensities, which is in agreement with dedicated machine studies in the AGS. Polarization also reduces with the increase in bunch intensity. We have to evaluate the gain or loss to the FOM of p-p operation from the increase in bunch intensity.

With the luminosity-derived transverse emittance, we can estimate the beam-beam parameter based on Eq. (4). The right plot of Fig. 9 shows the total beam-beam parameters with 2 collisional IPs versus the averaged bunch intensity for the Blue ring. These data are taken at 5 minutes into store for all the physics stores in the previous $250 / 255 \mathrm{GeV}$ p-p runs. From this plot, the beam-beam parameter increased with the 


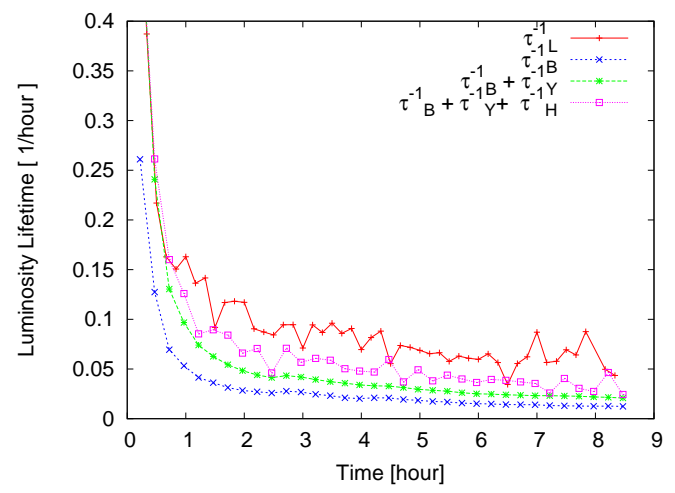

Figure 10: An example of luminosity decay rate and its contributions for Fill 16697.

bunch intensity. The typical total beam-beam parameter was 0.14 for the routine physics stores in the 2013 p-p run. There are a few stores in that year with initial bunch intensities larger than $1.9 \times 10^{11}$ but with lower beam-beam parameters, which will be discussed later.

\subsection{Luminosity Lifetime}

Mathematically, the evolution of luminosity at store can also be fitted with double exponentials,

$$
L(t)=L_{1} \exp \left(-t / \tau_{1}\right)+L_{2} \exp \left(-t / \tau_{2}\right) .
$$

Here $L_{1,2}$ and $\tau_{1,2}$ are the fitting parameters. $L_{1}$ and $\tau_{1}$ represent the early fast luminosity loss at the beginning of store. Typical $\tau_{1}$ is 1.5 hours. Together with the store turning around time, Eq. (21) is often used to determine the optimized physics store length to maximize the integrated luminosity.

According to Eq. (1), the luminosity decay rate is contributed by

$$
\begin{aligned}
\tau_{L}(t)^{-1} & =-\frac{1}{L(t)} \frac{d L(t)}{d t} \\
& =\tau_{B}^{-1}+\tau_{Y}^{-1}-\tau_{\langle\epsilon>}{ }^{-1}+\tau_{H}{ }^{-1} .
\end{aligned}
$$

Here $\tau_{B, Y}^{-1}$ are the particle loss rates in the Blue and Yellow rings. $\tau_{<\epsilon>}^{-1}$ is the transverse emittance growth rate, $\tau_{<\epsilon\rangle}^{-1}=-\frac{1}{\langle\epsilon\rangle(t)} \frac{d<\epsilon\rangle(t)}{d t} . \tau_{H}^{-1}$ is the growth rate of the hour-glass factor, $\tau_{H}^{-1}=-\frac{1}{H(t)} \frac{d H(t)}{d t}$.

As an example, Figure 10 shows the luminosity decay rate and its contributions for Fill 16697 . We calculated individual contribution rates every 15 minutes. For this fill, the instant luminosity reduced $40 \%$ in the first 2 hours. In the rest 6 hours at store, the averaged luminosity decay rate was $6 \% /$ hour.

According to Fig. 10, in the first hour of this store, the luminosity decay rate was dominated by the large particle loss rates in both rings. The bunch intensity lost about $18 \%$ in each ring. After 2 hours into the store, the intensity losses in both rings reduced to $1.5 \%$ /hour which contributed $3 \%$ /hour to the luminosity decay rate. The hour-glass effect and the transverse emittance growth contributed another $3 \% /$ hour to the luminosity decay rate. To improve the luminosity lifetime and increase the integrated luminosity, we should reduce the large beam loss at the beginning of store.

\section{Mechanisms and Modelings}

In this section we present the mechanisms for the particle loss, emittance growth, and bunch length increase in the routine physics stores in the previous RHIC p-p runs.

\subsection{Mechanism of Beam Loss}

\subsubsection{Correlation of Particle Loss}

We attempted to correlate the observed particle loss rates with the optics parameters such as tunes, chromaticities, and the beam parameters such as bunch intensities, transverse emittances. However, these correlations could not be applied to general cases, even though they might be able to explain the observations of beam loss and or emittance blow-up over a period of operation. 

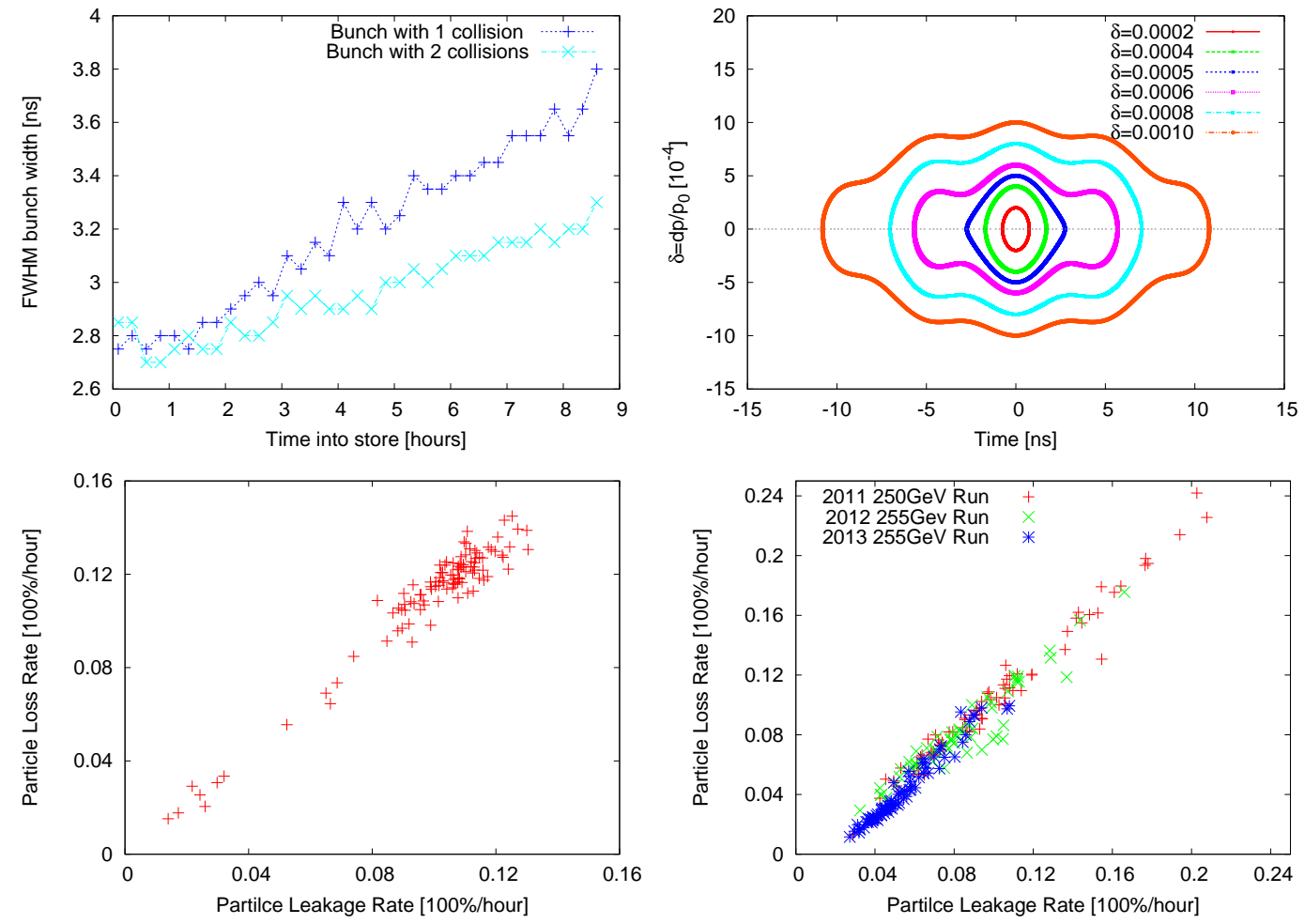

Figure 11: Top-left: Evolutions of bunch lengths for two adjacent bunches: one with 1 collision and the other with 2 collisions per turns. Top-right: The longitudinal phase space with $300 \mathrm{KV} 28 \mathrm{MHz} \mathrm{RF}$ cavity and $300 \mathrm{KV} 197 \mathrm{MHz}$ RF cavity at store. Bottom-left: Correlation between the particle loss percentage and the particle leakage percentage during the first hour of store for all bunches of Fill 16697. Bottom-right: Correlation between the particle loss percentage and the particle leakage percentage during the first hour of store for the beam in the Blue ring for all the physics stores in the past 250/255 GeV p-p runs. 
We normally observe that bunches with 1 collision per turn had a smaller particle loss rate at store than bunches with 2 collisions in the 2011 and 2012 p-p runs. In the following, we begin with comparing their longitudinal bunch profiles during the store. The top-left plot of Fig. 11 shows the bunch lengths for the two adjacent bunches as shown in the bottom-right plot of Fig. 6. The difference in their bunch lengths was very small at the beginning of store. After the first hour with collisions, the bunch lengths began to grow. However, the bunch length for the bunch with 1 collision per turn grew faster and got wider than the bunch with 2 collisions.

The top-right plot of Fig. 11 shows the longitudinal phase space with $360 \mathrm{KV} 28 \mathrm{MHz}$ and $300 \mathrm{KV}$ $197 \mathrm{MHz}$ RF voltages at the store energy $255 \mathrm{GeV}$. The horizontal axis is the distance to the center of the $28 \mathrm{MHz}$ RF bucket in units of ns. The central RF bucket of the $197 \mathrm{MHz}$ RF cavity is between [-2.5 ns, $2.5 \mathrm{~ns}]$. The vertical axis is the relative momentum deviation. The RF momentum acceptance for the combined RF systems is $1.1 \times 10^{-3}$. The typical longitudinal emittance of proton beam in RHIC is $2.0 \mathrm{eV}$.s at $250 \mathrm{GeV}$. The relative beam momentum spread is between $\pm 7 \times 10^{-4}$.

From the top-right plot of Fig. 11, the particles away from the center of $28 \mathrm{MHz}$ RF bucket have a large momentum deviation. Since the bunch with 1 collision per turn had a wider bunch width than the bunch with 2 collisions, it had more particles with larger momentum deviations than the bunch with 2 collisions. In other words, the bunch with 1 collision per turn had a larger off-momentum dynamic aperture than the bunch with 2 collisions per turn.

Comparing the longitudinal bunch profiles over the whole store, we notice that the particle intensity and particle distribution in the bunch tail did not change dramatically during store. However, the particle population in the central bunch area continuously decreased, especially in the first hour with collision.

Next we study the correlation between the particle loss and the particle movement in the longitudinal plane. With WCMs, we can continuously monitor each bunch's particle population and its longitudinal distribution. For instant, here we focus on the change of particle population in the central area $[-5 \mathrm{~ns}$, $5 \mathrm{~ns}]$. Note that this area was chosen arbitrarily. For particles between [-5ns, $5 \mathrm{~ns}$ ], their relative momentum deviation are between $\left[-6 \times 10^{-4}, 6 \times 10^{-4}\right]$. Choosing a different central region does not affect the following conclusion.

The bottom-left plot of Fig. 11 shows the percentage of particle lost and the percentage of particles moving out of the central area [-5ns, 5ns] during the first hour after collision for all bunches of Fill 16697. The particle leakage percentage is calculated from the population reduction in the area [-5 ns, $5 \mathrm{~ns}]$ normalized by the initial bunch intensity. The points in the bottom-left corner of the plot represent bunches with 1 collision per turn. From this plot, we find a strong linear correlation between the particle loss rates and particle leakage rates for all the bunches during the first hour with collision.

The above linear correlation between the particle loss and the particle leakage for all bunches also holds for the rest of store. Furthermore, if we use the averaged WCM bunch profile and the total beam intensity loss, a similar linear correlation also exists between the total particle loss and the total particle leakage for all the stores in one run. For an example, the bottom-right plot of Fig. 11 shows the correlation between the total particle loss and the total particle leakage percentages during the first hour of store for the Blue ring for all the physics stores in the past $250 / 255 \mathrm{GeV}$ p-p runs.

In the normal p-p operation, we did not observe de-bunched beam. Therefore, all the particle losses should originate from bunched beam and took place in the transverse plane instead of in the longitudinal plane through leaking out of the RF buckets. Limited by the transverse off-momentum aperture, the bunch length could not grow freely and an semi-equilibrium distribution of particles was formed at the bunch tail. As a result, the beam intensity loss rate was linearly correlated to the rate of particle leakage out of the bunch central area.

Since we did not observe large particle loss without collision, we conclude that the large particle loss in the previous RHIC p-p runs was due to a limited off-momentum dynamic aperture which was reduced by beam-beam interaction.

\subsubsection{Off-momentum Dynamic Aperture}

Here we calculate and compare the off-momentum dynamic apertures without and with beam-beam interaction. Dynamic aperture [35] is defined as the maximum betatron amplitude within which particles are not lost after a certain number of tracking turns. The long-term dynamic aperture converges to the boundary between regular and chaotic motion.

In the dynamic aperture calculation, we track particles in a 6 -dimensional phase space $\left(x, p_{x}, y, p_{y}, c \Delta t, \delta=\right.$ $\left.d p / p_{0}\right)$ up to $10^{6}$ turns with SimTrack [36, 37]. The initial particles are launched in 10 equally spaced direc- 


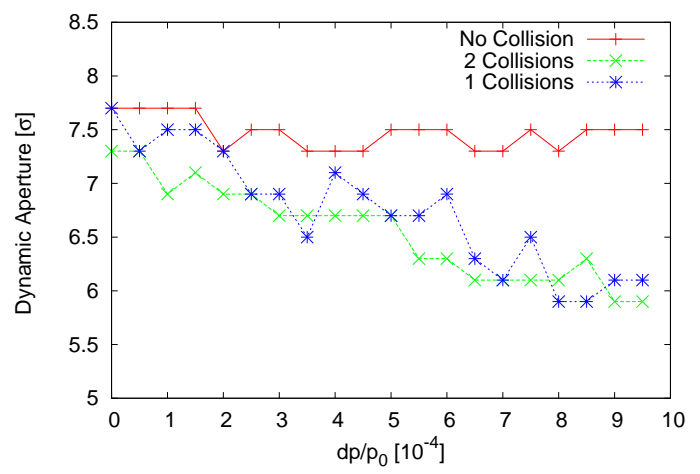

Figure 12: Calculated off-momentum dynamic apertures versus the relative momentum deviation $d p / p_{0}$ for the Blue ring. The lattice tunes are $(0.690,0.685)$ and $\beta^{*}=0.65 \mathrm{~m}$.

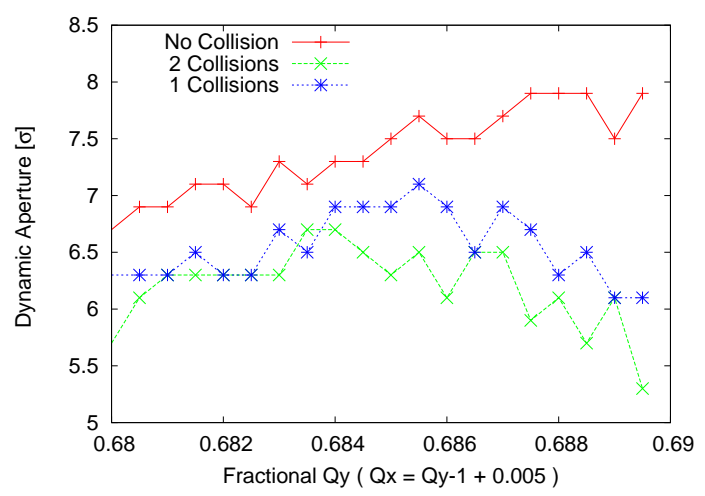

Figure 13: Calculated off-momentum dynamic apertures for the Blue ring in a tune scan along the diagonal in the tune space between $2 / 3$ and $7 / 10 . \beta^{*}=0.65 \mathrm{~m}$ at the collisional IPs.

tions in the first quadrant of the $\left(\sqrt{2 J_{x} \beta_{x}} / \sigma_{x}, \sqrt{2 J_{x} \beta_{y}} / \sigma_{y}\right)$ plane, $\beta_{x, y}$ are the $\beta$-functions at the starting point of the tracking. The dynamic aperture is given in units of the transverse rms beam size $\sigma$. We only compare the minimum dynamic apertures among these angles between different beam-beam conditions.

The lattices for the $250 / 255 \mathrm{GeV}$ p-p runs are used for this study. The $\beta^{*} \mathrm{~s}$ at the collision points are $0.65 \mathrm{~m}$. Based one the offline optics models, the second order chromaticities are $(2100,4400)$ for the Blue ring and $(-6100,-1700)$ for the yellow ring. Second order chromaticities are defined as $\xi_{x, y}^{(2)}=\frac{1}{2} \frac{\partial^{2} Q_{x, y}}{\partial \delta^{2}}$.

Figure 12 shows the off-momentum dynamic apertures versus the relative momentum deviation $d p / p_{0}$ for the Blue ring. Three cases are studied: without collision, with 2 collisions at IP 6 and IP8, and with 1 collision at IP6. The particle energy is $255 \mathrm{GeV}$. The lattice tunes are $(28.690,29.685)$, which are close to the normal operational settings. The linear chromaticities are corrected to $(1,1)$ before tracking. $360 \mathrm{KV}$ $28 \mathrm{MHz}$ and $300 \mathrm{KV} 197 \mathrm{MHz}$ RF voltages are used. The normalized rms transverse emittance is $3.3 \mu \mathrm{m}$. The bunch intensity is $1.8 \times 10^{11}$, which gives a total beam-beam parameter of 0.0135 .

From Fig. 12, the dynamic aperture without collision is around $7.5 \sigma$ for $d p / p_{0}$ between 0 and $1.0 \times 10^{-3}$. Beam-beam interaction reduces the dynamic aperture with collisions. The larger momentum deviation is, the smaller dynamic aperture is. For a $d p / p_{0}$ of $7 \times 10^{-4}$, the dynamic aperture with collision is around $6 \sigma$, which is $1.5 \sigma$ less than the case without collision. And for most momentum errors shown in Fig. 12, the dynamic aperture with 1 collision is slightly larger than the one with 2 collisions.

Figure 13 shows the off-momentum dynamic apertures for the Blue ring without and with beam-beam interactions in a tune scan below the diagonal in the tune space $2 / 3$ and $7 / 10$. The horizontal axis is the vertical fractional lattice tune. The horizontal fractional tune is always 0.005 higher than the vertical one. The same beam parameters as the last dynamic aperture calculation are used. The initial relative momentum deviation is set to $6 \times 10^{-4}$, which is about 3 times the rms relative momentum deviation.

From Fig. 13, the case without collision always gives the largest dynamic aperture among the three cases. For the cases with beam-beam interaction, when the vertical tune is between 0.684 ans 0.690 as the routine physics stores, the case with 1 collision per turn gives a larger dynamic aperture than the case with 
2 collisions per turn.

To summarize, analysis of operational data shows that the particle loss in the previous RHIC p-p operation is caused by the limited transverse off-momentum dynamic aperture. Numeric simulations verify that beambeam interaction reduces off-momentum dynamic aperture. And bunches with 1 collision per turn have a slightly larger dynamic aperture than bunches with 2 collisions.

\subsection{3 $197 \mathrm{MHz}$ RF Cavity Voltage}

Here we discuss the role of $197 \mathrm{MHz}$ RF voltage. As mentioned above, it is used to reduce the bunch length so that there are more collisions in the central areas of detectors. For narrow vertex physics programs, only collisions in the central area [-10 cm, $10 \mathrm{~cm}]$ are interested.

With $300 \mathrm{KV}$ RF voltage in the 2011 and 2012 p-p runs, the rms bunch length was $0.41 \mathrm{~m}$ at the beginning of store. With $100 \mathrm{KV}$ RF voltage in 2013, it was $0.61 \mathrm{~m}$. With the longitudinal bunch profiles from WCMs, we are able to calculate the luminosity reduction factor due to hour-glass effect and the percentage of collisions in the central area.

The luminosity reduction factor from hour-glass effect was 0.72 at the beginning of store and 0.64 at 6 hours into store for both 2012 and 2013 runs. There was no increase in the hour-glass factor with a higher $197 \mathrm{MHz} \mathrm{RF}$ voltage. Right after collision, the percentage of collisions in the [-10 cm, $10 \mathrm{~cm}]$ area was 0.66 in 2012 and 0.60 in 2013. After 6 hours into store, the percentage dropped to 0.56 in 2012 and 0.51 in 2013. There were 10\%- 15\% more collisions in the central area in 2012 than that in 2013.

As shown in the up-right plot of Fig. 6, the particle loss rate in 2012 was more than double that in 2013. A higher $197 \mathrm{MHz}$ RF cavity voltage creates a smaller RF bucket area in the bunch center and a larger beam momentum spread. With $300 \mathrm{KV}$ RF voltage, a large portion of particles could not be fitted in the central $197 \mathrm{MHz}$ RF bucket, as shown in the bottom-left plot of Fig. 7. These particles in the bunch tail have a large momentum deviation. When the bunches were brought into collision, they would get lost in transverse plane sooner or later due to a reduced transverse off-momentum dynamic aperture.

Another reason for the larger beam loss with $300 \mathrm{KV} 197 \mathrm{MHz}$ RF voltage is that the particle density at the bunch center was higher. As to be discussed later, several diffusion processes can move particles out of the central bunch area. The higher the particle intensity is, the larger diffusion rate is.

\subsection{Mechanisms of Emittance Growth}

\subsubsection{Beam-Gas Scattering}

Interactions between the stored protons and the residual gas includes inelastic and elastic interactions. For the RHIC p-p operation, the most important beam-gas interactions are the inelastic nuclear collisions and the elastic nuclear Coulomb scatterings between the protons and the nuclei of gas atoms $[38,39]$. The inelastic nuclear collisions cause proton loss and reduce beam lifetime, while the multiple elastic nuclear Coulomb scatterings cause proton beam emittance growth.

The cross section of the nuclear collision can be estimated as [40]

$$
\sigma_{N} \simeq \pi R_{N}^{2},
$$

with $R_{N}=r_{0}\left(A_{i}^{1 / 3}+A_{t}^{1 / 3}\right), r_{0} \simeq 1.2 \mathrm{fm}, A_{i, t}$ are the atom mass numbers of the projectile ions and the target gas atoms. The cross sections between proton and the residual gas atoms are in an order of $10^{-25} \mathrm{~cm}^{-2}$.

In the normal RHIC operation, $83 \%$ of the RHIC beam pipe is at $4.5 \mathrm{~K}$ with a pressure of $0.01 \mathrm{nTorr}$ and the rest at the room temperature $300 \mathrm{~K}$ with a pressure of 0.5 nTorr. The warm section are mainly in the six IRs. The residual gases are made of $100 \% \mathrm{H}_{e}$ gas in the cold region, and $95 \% \mathrm{H}_{2}$ and $5 \% \mathrm{CO}$ in the warm region. Based on the RHIC vacuum condition, the beam-gas lifetime in the RHIC p-p operation is 1250 hours and is negligible.

The cross section of single elastic Coulomb scatterings between protons and the nuclei of gas atoms is give by Rutherford cross section [41]. The averaged square of scattering angle $\theta$ is

$$
<\theta^{2}>=2 \theta_{\min }^{2} \ln \left(\frac{\theta_{\max }}{\theta_{\min }}\right)=4 \theta_{\min }^{2} \ln \left(204 Z_{t}^{-1 / 3}\right),
$$

where $\theta_{\min }$ is the minimum scattering angle due to screening of electrons of target atoms, $\theta_{\max }$ the maximum scattering angle due to finite sizes of target nuclei, $Z_{t}$ the atom number of the target atom. For the RHIC 
p-p operation, $\sqrt{<\theta^{2}>}$ is in an order of $10^{-8}-10^{-7} \mathrm{rad}$. The rms angle spread $\sigma_{x^{\prime}}$ of the proton beam is in an order of $10^{-5}$ rad. Therefore single elastic Coulomb scattering can not cause proton loss.

The growth rate of the rms normalized emittiace of the proton beam due to multipole elastic Coulomb scatterings between protons and the nuclei of gas atoms is calculated with [42]

$$
\frac{d \epsilon_{n, r m s}}{d t}=2 \pi \gamma_{i}<\beta>n_{t} \beta_{i} c\left(\frac{2 Z_{i} Z_{t} r_{p}}{A_{i} \beta_{i}^{2} \gamma_{i}}\right)^{2} \ln \left(204 Z_{t}^{-1 / 3}\right)
$$

where $\beta_{i} c$ and $\gamma_{i}$ are the velocity and Lorentz factor of the projectile ions, $n_{t}$ the density of gas atoms. $\langle\beta>$ is the averaged $\beta$ function along the ring, which is about $45 \mathrm{~m}$ in the cold sections and $115 \mathrm{~m}$ in the warm sections.

For the $100 \mathrm{GeV}$ RHIC p-p operation, the calculated normalized rms emittance growth due to multiple elastic Coulomb scatterings is $4.9 \times 10^{-3} \mu \mathrm{m}$ /hour. And for the $250 / 255 \mathrm{GeV}$ RHIC p-p operation, it is $2.5 \times 10^{-3} \mu \mathrm{m} /$ hour. Comparing to the proton emittance in RHIC, the emittance growth due to beam-gas interaction can be neglected.

\subsubsection{Touschek Effect}

Coulomb scattering of charged particles in a bunched beam results in an exchange of energy between different degrees of freedom [43]. Usually two scattering regimes are considered: the single scattering when a rare single large-angle scattering makes a large change to particle momentum ( Touschek effect $[44,45,46,47]$ ), and the multiple small-angle scatterings which lead to a diffusion process (IBS effect $[48,49,50]$ ). Touschek effect can cause particle loss and create a non-Gaussian bunch tail. IBS is usually responsible for the changes of particle distribution in the bunch core.

The particle loss rate $\tau_{T}^{-1}\left(\delta_{m}\right)$ due to Touchek effect for a 3-d Gaussian distribution is given in Ref. [43]. $\delta_{m}$ is the maximum momentum aperture, which is assumed to be much larger than the beam's momentum spread. Here we use these equations to estimate the particle leakage rate out of the central bunch area due to Touschek effect. For an example, we choose the central area as [-5 ns, $5 \mathrm{~ns}] .5 \mathrm{~ns}$ offset from the bunch center gives a maximum relative momentum deviation $6 \times 10^{-4}$.

Considering the fact that particles with different initial momentum deviation need different momentum change to leak out of the central bunch area, the total leakage rate is given by [51]

$$
\tau_{\text {leakage }}^{-1}=\int_{0}^{6 \times 10^{-4}} \tau_{T}^{-1}\left(6 \times 10^{-4}-\delta\right) \rho(\delta) d \delta .
$$

$\rho(\delta)$ is the distribution of relative momentum deviation for particles in the area [-5 ns, $5 \mathrm{~ns}]$.

For simplicity, we assume a Gaussian distribution of particles in the bunch central area. For Fill 16697, the bunch intensity is $1.6 \times 10^{11}$. However, only about $1.2 \times 10^{11}$ particles are in the central Gaussian distribution. The rms bunch length was $0.4 \mathrm{~m}$, and the relative rms momentum deviation was $2 \times 10^{-4}$. Figure 14 shows $\tau_{T}^{-1}$ as a function of $\delta_{m}, \delta_{m}$ is scanned from $1.0 \times 10^{-5}$ to $6 \times 10^{-6}$ with a step size of $1.0 \times 10^{-5}$.

According to Eq. (26), the calculated particle leakage rate out of the central area [-5ns, $5 \mathrm{~ns}]$ is about $1 \%$ /hour, which is much less than the actual observations. As we reported earlier, more than $10 \%$ of total particles leaking out of the central area [-5 ns, $5 \mathrm{~ns}]$ during the first hour of store in the $2012250 \mathrm{GeV}$ run. Therefore, Touschek effect is not the main cause to move particle out of the central bunch area.

\subsubsection{IBS Effect}

The exact equations to calculate the IBS growth rates for a 3-d Gaussian distribution bunch are given in Ref. [43]. For a quick estimate, we use an approximate approach given in Ref. [52]. The difference in the growth rates between the exact and approximate calculations is within 20\%. The IBS growths rates from the approximate approach are given by

$$
\begin{gathered}
\tau_{I B S, \|}^{-1}=\frac{1}{\sigma_{p}^{2}} \frac{d \sigma_{p}^{2}}{d t}=\frac{r_{i}^{2} c N_{i} \Lambda}{\sqrt{\pi / 2} \beta \gamma^{3} \epsilon_{x}^{3 / 2}<\beta_{x}^{1 / 2}>\sigma_{l} \sigma_{p}^{2}}, \\
\tau_{I B S, x, y}^{-1}=\frac{1}{\epsilon_{x, y}} \frac{d \epsilon_{x, y}}{d t}=\frac{1}{2} \frac{\sigma_{p}^{2}}{\epsilon_{x}}<\frac{H_{x}}{\beta_{x}}>\tau_{\|}^{-1} .
\end{gathered}
$$




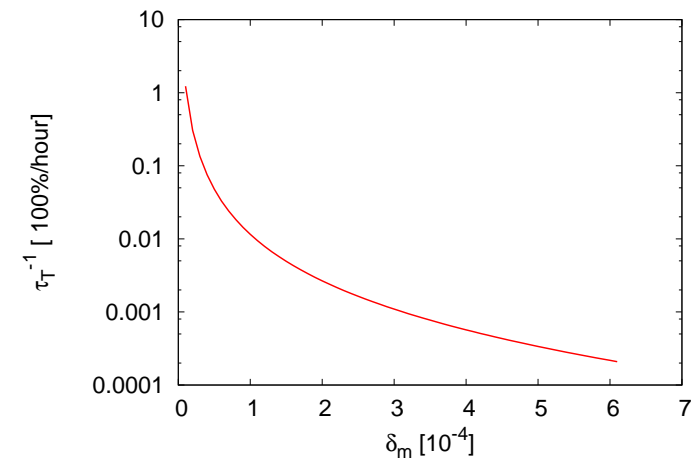

Figure 14: The precentage of protons per hour having relative momentum change $\delta_{m}$ due to Toucheck effect.

Here $\sigma_{p}$ is the rms relative momentum spread, $\sigma_{l}$ is the rms bunch length, and $\epsilon_{x}$ is the transverse normalized rms emittance. $r_{p}$ is the classic radius of proton. $\Lambda$ is the Coulomb logarithm. $H_{x}=\left(D_{x}^{\prime} \beta_{x}+\alpha_{x} D_{x}\right)$ is the invariant of dispersion, $D_{x}$ is horizontal dispersion, $\alpha_{x}$ is the horizontal alpha-function of betatron motion. In Eq. (28), we assumed same emittance growth rates for the horizontal and vertical planes, which is the case for the normal RHIC operation.

For the RHIC p-p operation lattices with $\beta^{*}=0.65 \mathrm{~m}$ at IP6 and IP8, we have $<\beta_{x}>=6.7 \mathrm{~m}$ and $<H_{x} / \beta_{x}>=0.045$. With the following initial parameters at the beginning store: bunch intensity $1.7 \times 10^{11}$, rms normalized emittance $2.5 \mathrm{um}$, rms bunch length $0.4 \mathrm{~m}$, and rms relative momentum spread $1.4 \times 10^{-4}$, the IBS growth times are

$$
\begin{aligned}
\tau_{I B S, \|} & =1.0 \text { hours } \\
\tau_{I B S, x, y} & =20.2 \text { hours } .
\end{aligned}
$$

The longitudinal IBS growth is much faster than the transverse emittance. For an example, if there is no particle loss in the first hour at store, the momentum spread and the bunch length would increase $40 \%$ while the transverse emittance increases $3 \%$.

Next we estimate the percentage of particles moving out of central bunch area during the first hour at store. From the longitudinal bunch profile measurements, such as shown in the bottom-left plot of Fig. 7, the particle distribution and population out of the [-2.5 ns, $2.5 \mathrm{~ns}]$ area did not change dramatically during the first hour at store.

Knowing the rms bunch sizes at the beginning of store, if we assume that there is no particles loss to the central Gaussian particle distribution and its bunch length can grow freely, we estimate that there would be about $11 \%$ of total particles moving out of the area [-2.5 ns, $2.5 \mathrm{~ns}]$ due to IBS effect during the first hour at store. This is in a good agreement with the observed particle leakage rate.

\subsubsection{Modeling of Emittance and Bunch Length}

In the following, we numerically model the transverse emittance and bunch length growths at store only with IBS effect. The initial bunch length, transverse emittance, and bunch length are to be used. Assuming a 3-d Gaussian distribution for the particles in the bunch center, we calculate the instantaneous IBS growth rates with Eqs. (25) and (26). The real evolution of bunch intensity throughout the store is used. In the calculation, we update the IBS growth rates, transverse emittance, and bunch length every 20 seconds .

First we model the bunch length growth without beam-beam interaction. As an example, Fig. 15 shows the measured and IBS-predicted rms bunch lengths for the beam in the Yellow ring for Fill 16715. This store was used for the proton polarization lifetime measurement at store and there was no collision. The voltage of the $197 \mathrm{MHz}$ RF cavity was $60 \mathrm{KV}$. The bunch profile was Gaussian.

The initial bunch intensities was $0.8 \times 10^{11}$, the rms bunch length was $0.56 \mathrm{~m}$, and the rms relative momentum spread was $2 \times 10^{-4}$. A typical normalized rms transverse emittance $3 \mu \mathrm{m}$ at the beginning of store was assumed since we did not have a good IPM emittance measurement for this store. The beam loss rate was less than 1\%/hour at store except during polarization measurement. From Fig. 15, IBS basically reproduces the measured bunch length growth. There were two small jumps in the measurement data which was caused by polarization measurements.

Next we numerically model the emittance and bunch length growths for Fill 16697 with collisions. As discussed above, the reductions in emittance and bunch length at the beginning of store were related to a 


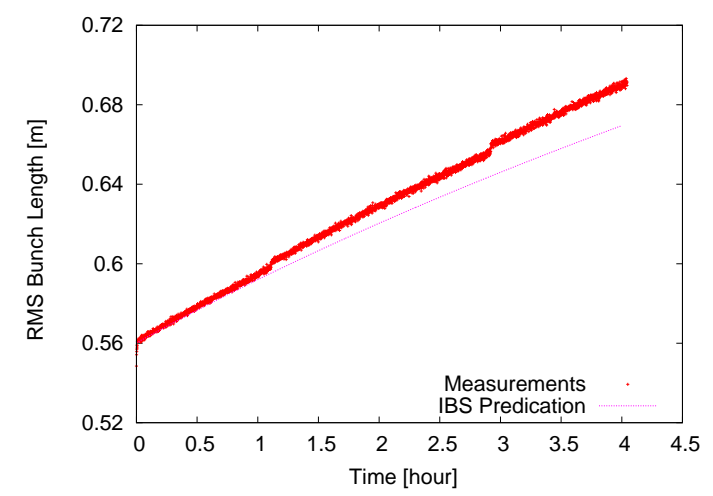

Figure 15: Numerical modeling of bunch length growth without collision at store. Fill 16715 is used.

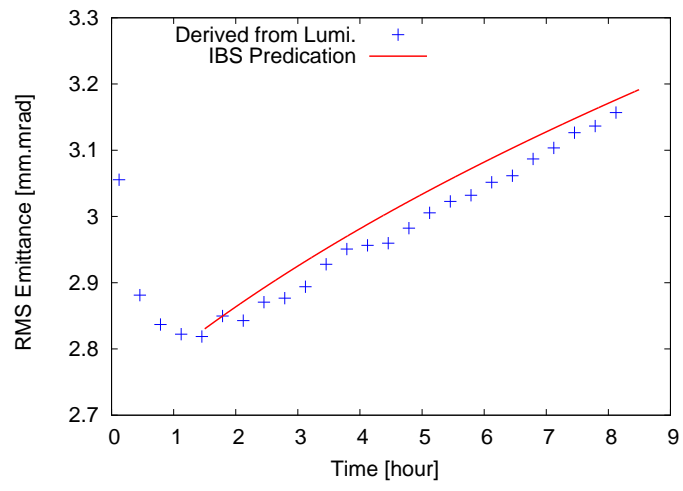

Figure 16: Numeric modeling of emittance growth with collision at store. Fill 16697 is used.

fast large intensity loss. Our modeling starts 1.5 hours into store and the real bunch intensity evolution is used. Figures 16 and 17 show the IBS-predicted transverse emittance and rms bunch length growths for the beam in the Blue ring against their actual measurements.

In this calculation, we still used a 3 -d Gaussian distribution for the particles in the bunch central area. The initial bunch intensity is $1.1 \times 10^{11}$, the rms bunch length is $0.42 \mathrm{~m}$, and the initial relative rms momentum spread is $3.5 \times 10^{-4}$. The initial normalized rms transverse emittance is $2.8 \mu \mathrm{m}$, which is derived from luminosity. In the 7-hour-long store, the actual emittance grew $11.5 \%$ and the bunch length grew $23.8 \%$. From Figs. 16 and 17, IBS largely reproduces the actual emittance and bunch length growths.

To summarize, based on the above calculations of particle loss rates and emittance growth rates from beam-gas interaction, Touschek effect, and IBS effect, we conclude that the IBS effect is the main cause to move particles out of the central bunch area. Numeric modelings of transverse emittance and bunch length growths with IBS effect largely reproduce the real measurements without and with collision.

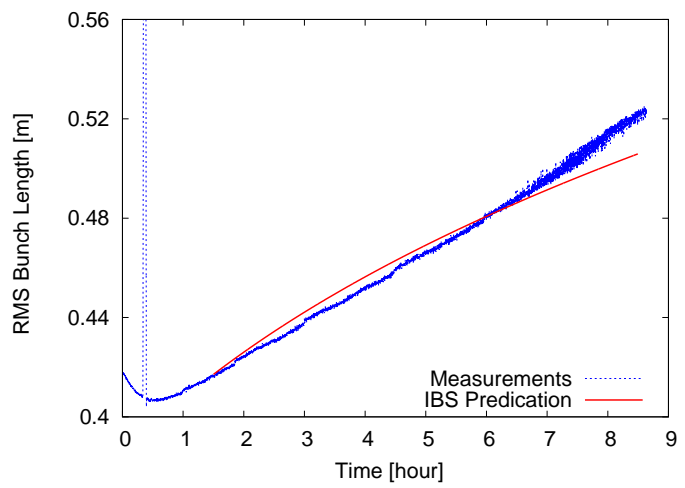

Figure 17: Numeric modeling of bunch length growth with collision at store. Fill 16697 is used. 


\section{Challenges and Limitations}

In the section we will present beam-beam interaction related operational challenges and their remedies in the previous RHIC p-p operation. Head-on beam-beam compensation with electron lenses to reduce the large beam-beam tune spread in RHIC is also discussed briefly.

\subsection{IR Multipole Field Errors}

Magnetic nonlinear field errors in the RHIC IRs play a significant role in the reduction of dynamic aperture with beam-beam interaction. These IR nonlinearities come from the magnetic imperfections in the triplet quadrupoles and the separation dipoles. Due to the tight installation schedule, only some of those magnets were measured cold. Even when the cold measurements are available, the geometric configuration of the leads was altered during the magnet assemblies in the RHIC tunnel. To build the nonlinear IR model, we had to scale the power supply currents from the magnet measurement condition to the real operating condition. If the measurement data are only available at room temperature, a warm to cold conversion had to been used $[53,54]$.

A task force was set up in 2003 to study the effects of IR nonlinearities on the proton dynamic aperture with beam-beam interaction. By blocking one or several orders of IR nonlinear field errors, we attempted to search the predominant multipole components to the dynamic aperture reduction [53]. Simulation results show that there is no single predominant multipole component, even though removing the sextupole components gives a slightly larger dynamic aperture in the vicinity of third order resonances.

Some nonlinear correcting spool pieces were integrated in the triplet quadrupoles during manufacture. However, only some low order nonlinear correctors in IR6 and IR8 have been equipped with individual power supplies. IR bump method is used on line to correct the sextupole, skew sextupole, and octupole components in IR6 and IR8 $[55,56]$. By creating a local orbit bump across one IR, we managed to minimize the tune shifts from the feed-down of multipole errors with the local IR nonlinear correctors. The correction strengths for the local IR sextupoles, skew setupoles, and octupoles are always installed in the RHIC operation. These strengths found from the online IR bump method and the action-angle minimization [57] based on the offline nonlinear IR model agreed well.

During the $100 \mathrm{GeV}$ RHIC polarized proton run in 2009, 10- and 12-pole interaction region correctors were used operationally for the first time [58]. 10-poles are the second allowed harmonic error in dipoles, and 12-poles are the first allowed harmonic in quadrupoles. The correctors were set with an automatic scanning procedure during physics stores that adjusted their strengths to minimize the beam loss rate. Simulations show an increase of dynamic aperture by $8 \%$ with these experimentally found 10- and 12-pole corrector strengths. The actual integrated luminosity per store increased by $4 \%$.

In 2009, we collided protons at $100 \mathrm{GeV}$ and $250 \mathrm{GeV}$ with a same $\beta^{*}=0.7 \mathrm{~m}$. The proton bunch intensities, the normalized transverse emittances, and therefore the total beam-beam parameters were almost same too. However, in the operation, we observed a much shorter beam lifetime in the $100 \mathrm{GeV}$ run than that in the $250 \mathrm{GeV}$ run. Simulation shows that the dynamic apertures in units of $\mathrm{mm}$ only differ by $5 \%$ at 100 and $250 \mathrm{GeV}$. However, in units of rms beam sizes, the dynamic aperture at $250 \mathrm{GeV}$ is $52 \%$ larger than that at $100 \mathrm{GeV}$. Considering the fact that the proton rms beam size at $250 \mathrm{GeV}$ is smaller than at $100 \mathrm{GeV}$, a particle with a same amplitude in $\sigma$ s at $250 \mathrm{GeV}$ would sample smaller IR nonlinear fields [59].

\subsection{Chromatic Effects}

To increase the luminosity, we reduced $\beta^{*}$ s at the collisional IPs. However, a low $\beta^{*}$ lattice increases the nonlinear chromaticities and reduces the off-momentum dynamic aperture.

Figure 18 shows the fitting parameters $\tau_{1}$ and $\tau_{2}$ for the luminosity evolution at store for all the physics stores in the 2008, 2009, and $2012100 \mathrm{GeV}$ RHIC p-p runs. The $\beta^{*} \mathrm{~s}$ at the collisional IPs were $1.0 \mathrm{~m}, 0.7 \mathrm{~m}$, and $0.85 \mathrm{~m}$ for the three years respectively. $\tau_{2}$ mainly represents the luminosity lifetime after the first hour into store. The averaged $\tau_{2}$ s are 17 hours for the 2008 run, 11 hours for the 2009 run. Based on the offline dynamic aperture calculation, we increased $\beta^{*}$ to $0.85 \mathrm{~m}$ in the $2012 \mathrm{run}$ and the averaged $\tau_{2}$ increased to 17 hours.

Figure 19 shows the calculated dynamic apertures with beam-beam interaction versus $\beta^{*}$ s at IP6 and IP8. The particle energy is $100 \mathrm{GeV}$. The tunes without collision are $(28.688,29.685)$. The proton bunch intensity is $1.5 \times 10^{11}$. The transverse emittance is $3.3 \mu \mathrm{m}$. The total beam-beam parameter is 0.011 . The initial relative momentum deviation of particles is $12.5 \times 10^{-4}$, which is 3 times the rms relative momentum 


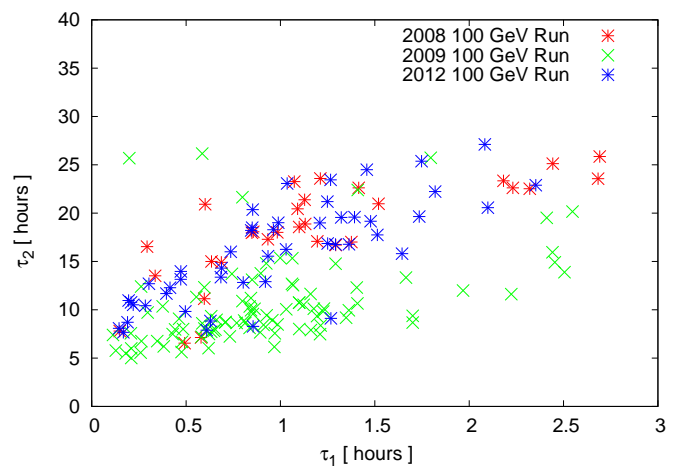

Figure 18: Fitting parameters of luminosity evolution $\tau_{1}$ and $\tau_{2}$ for all the physics stores in the 2008, 2009, and 2012100 GeV RHIC p-p runs.

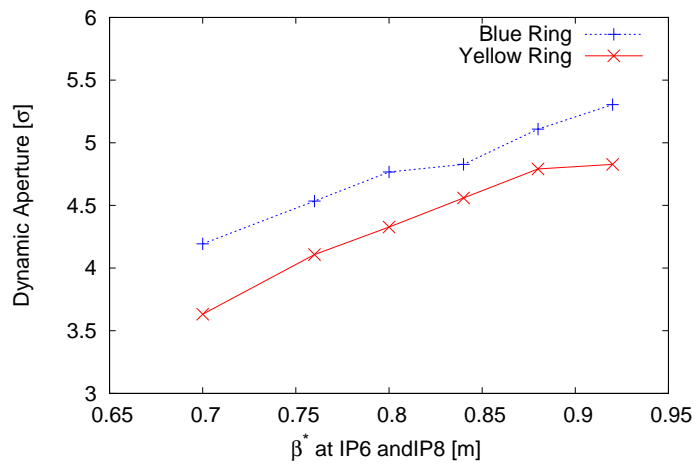

Figure 19: Calculated dynamic apertures with beam-beam interaction versus the $\beta^{*}$ values at the collisional IP6 and IP8. The particle energy is $100 \mathrm{GeV}$.

deviation. From this plot, the dynamic aperture with beam-beam interaction drops as $\beta^{*}$ decreases. With $\beta^{*}=0.7 \mathrm{~m}$, the calculated dynamic apertures are $4.2 \sigma$ in the Blue ring and $3.6 \sigma$ in the Yellow ring. With a larger $\beta^{*}=0.85 \mathrm{~m}$, they increase to $4.7 \sigma$ and $4.5 \sigma$ respectively.

Based on the perturbation theory, the second order chromaticities are

$$
\begin{aligned}
\xi_{x, y}^{(2)}= & -\frac{1}{2} \xi_{x, y}^{(1)}+\frac{1}{8 \pi} \oint\left[\mp K_{1} \pm K_{2} D_{x}\right] \frac{\partial \beta_{x, y}}{\partial \delta} d s \\
& +\frac{1}{8 \pi} \oint \pm K_{2} \beta_{x, y} D_{x}^{(2)} d s .
\end{aligned}
$$

where $D_{x}=\frac{\partial x_{c o}}{\partial \delta}, D_{x}^{(2)}=\frac{\partial^{2} x_{c o}}{\partial \delta^{2}}$. Based on Eqs. (29), as an example, Figure 20 shows the contributions from all quadrupoles and sextupoles to the horizontal second order chromaticities for the Yellow ring lattice with $\beta^{*}=0.65 \mathrm{~m}$. The starting point of the horizontal axis is IP12. The triplet quadrupoles in the two low- $\beta^{*}$ IRs contribute $80 \%$ of the total second order chromaticities [60].

The nonlinear chromaiticities can be corrected globally in RHIC. For each ring, there is a total of 24 independent sextupole power supplies. In each arc, we split the focusing and defocusing sextupoles into two sub-families. Therefore, there are 4 sub-families of sextupoles in each arc. They are nested as (SF1, SD1, SF2, SD2,SF1, SD1, SF2, SD2,...). Different correction methods and optimization algorithms have been used for second order chromaticity correction in RHIC [61].

Besides chromatic correction for the existing RHIC p-p lattices, we are also exploring new lattices to provide a large off-momentum dynamic aperture. Since the non-linear chromaticities are mainly contributed by the triplet quadrupoles in the two low- $\beta^{*}$ IRs, we can adjust the phase advances between IP6 and IP8 to be $(p+1 / 2) \pi, p$ is an integer, to minimize $d \beta / d \delta$ in these regions [62]. Other approaches, such as the achromatic-telescopic-squeezing (ATS) scheme [63] adopted for the HL-LHC program with exact $90^{\circ}$ FODO cells in the arcs to cancel the third order RDTs and $\pi / 2$ phase advance between the first sextupole and the triplet quadrupole to cancel the second order chromaticities are also being studied. 


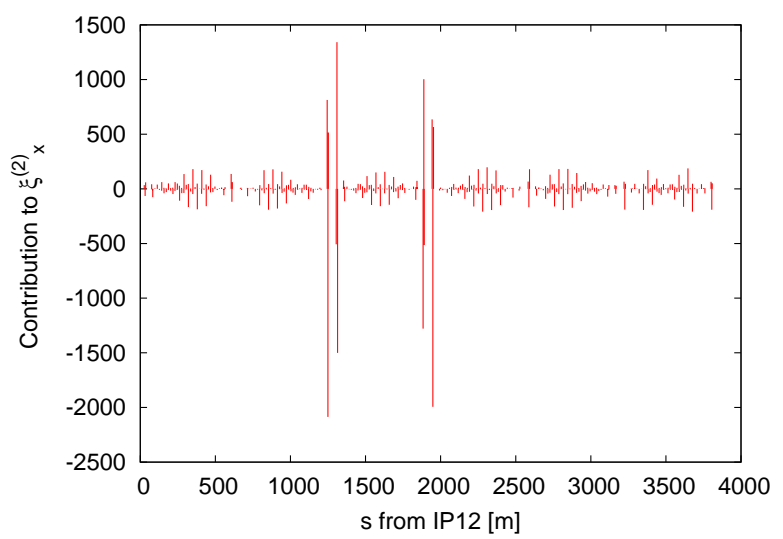

Figure 20: Contributions of quadrupoles and sextupoles along the ring to the global horizontal second order chromaticity. The $2012100 \mathrm{GeV}$ Yellow ring lattice is used. $\beta^{*} \mathrm{~s}$ at IP6 and IP8 are $0.85 \mathrm{~m}$.

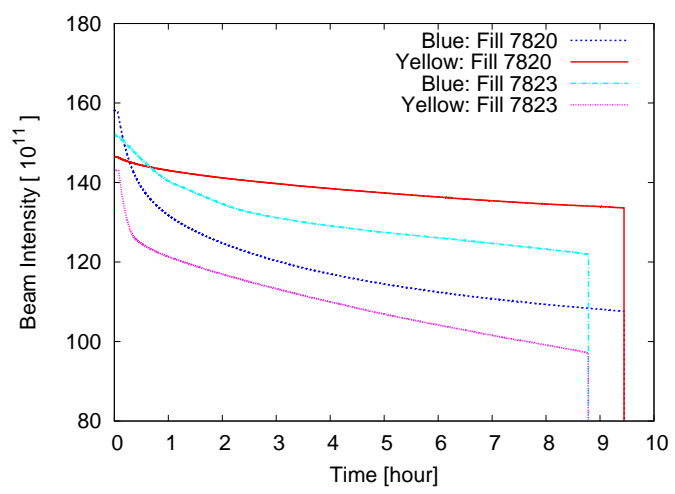

Figure 21: Evolutions of beam intensities from two consecutive stores in the $2006100 \mathrm{GeV}$ p-p run. The horizontal and vertical lattice tunes will be put into legends.

\section{3 $3 Q_{x, y}$ Resonances}

Beam-beam interaction introduces an amplitude-dependent tune which is proportional to the bunch intensity. In the limited tune space between $2 / 3$ and $7 / 10$, a large beam-beam parameter will push particles in the bunch core towards the third order resonances at $Q_{x, y}=2 / 3$.

Even with a low beam-beam parameter in the $2006100 \mathrm{Gev}$ p-p run, we already noticed that a working point above the diagonal gave a worse beam lifetime with collisions than the one below the diagonal. Figure 21 shows the evolutions of beam intensities of two consecutive stores with swapped horizontal and vertical set tunes. The ring with a working point above the diagonal always had a larger beam loss. Since then, we always place the working points of both rings below the diagonal.

Figure 22 shows the calculated dynamic aperture with beam-beam interaction in a tune scan along the diagonal in the tune space between $2 / 3$ and $7 / 10$. The $\beta^{*} \mathrm{~s}$ at IP6 and IP 8 are $1.0 \mathrm{~m}$, which is the same as in 2006. The bunch intensity is $1.5 \times 10^{11}$ and the transverse rms emittance $3.3 \mu \mathrm{m}$. The initial relative momentum deviation is $12.5 \times 10^{-4}$. The difference between the fractional horizontal and vertical tunes are kept to 0.005 . The horizontal axis is the lower tune value, which can be the horizontal or the vertical tune depending on the working point is above or below the diagonal. Simulation results show that the dynamic aperture drops when the working point is close to the third order resonances. Near the third order resonances, a working point below the diagonal gives a larger dynamic aperture than the one above the diagonal.

With beam-beam interaction, $3 Q_{x, y}$ resonances not only reduce the beam lifetime but also increase the transverse emittances. With a large bunch intensity and a large beam-beam parameter in the $2013255 \mathrm{Gev}$ p-p run, limited by the available tune space, we had to place the beam-beam tune footprint lower close to the third order resonance to maintain the proton polarization. Operational experiences show that a vertical tune higher than 0.686 would reduce the polarization lifetime. Since the beam-beam tune footprint was close 


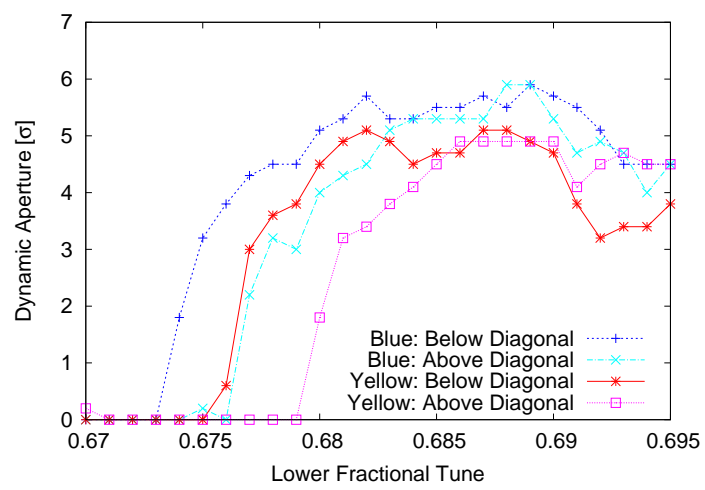

Figure 22: Calculated dynamic aperture in tune scans above and below the diagonal line. The horizontal tune is the lower lattice tune. The difference between the transverse fractional tunes is kept to 0.005 in the tune scan.

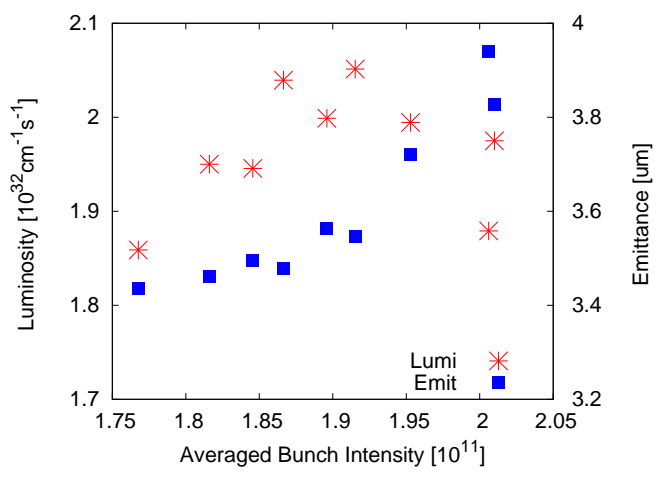

Figure 23: Luminosities and the luminosity-derived emittances in the $2013250 \mathrm{GeV}$ p-p run when we pushed the proton bunch intensities up to $2.0 \times 10^{11}$. Fill numbers are from 17415 to 17436 .

to the third order resonances, we normally observed transverse emittance blow-ups right after collision in the routine physics stores in 2013.

Figure 23 shows the luminosity and the luminosity-derived emittances when we pushed the proton bunch intensity beyond $1.85 \times 10^{11}$ in 2013 . Each point in Fig. 23 represents one store. The horizontal axis is the averaged bunch intensity between the Blue and Yellow rings. These data are taken at 5 mins after collision.

The lattice tunes without beam-beam interaction were $(0.688,0.685)$. Assuming a typical total beambeam parameter 0.014 , the tunes of particles in the bunch core were pushed by the beam-beam interaction down to $(0.674,0.671)$. From Fig. 23 , when the bunch intensity is beyond $1.85 \times 10^{11}$, we observed emittance blow-up and reduced luminosity. The reason is that the third order resonances blew up the emittances when a large beam-beam interaction pushes the tune footprint down close to $2 / 3$.

The $3 Q_{x, y}$ resonance RDTs are defined as

$$
\begin{aligned}
h_{3000} & =-\frac{1}{24} \sum_{i=1}^{N}\left(K_{2} L\right)_{i} \beta_{x, i}^{3 / 2} e^{i 3 \mu_{x, i}} \\
h_{0003} & =-\frac{1}{24} \sum_{i=1}^{N}\left(K_{2 s} L\right)_{i} \beta_{y, i}^{3 / 2} e^{i 3 \mu_{y, i}} .
\end{aligned}
$$

Here $\left(K_{2} L\right)$ and $\left(K_{2 s} L\right)$ are the integrated strengths of the sextupoles and skew sextupoles. Based on the offline optics model, the main sources for the $3 Q_{x, y}$ resonance RDTs are localized in IR 6 and IR 8 where $\beta \mathrm{s}$ are much larger than other regions. Without beam-beam interaction, we observed a large beam loss larger than $40 \%$ /hour when either tune was lower than 0.680. 3Q $Q_{x}$ resonance RDT was measured in RHIC with AC dipole excitations [64, 65].

In the RHIC operation, we normally use the local IR sextupole and skew sextupole correctors for the global $3 Q_{x, y}$ resonance corrections and use the sextupoles in the arcs for the nonlinear chromaticity correction [66]. 


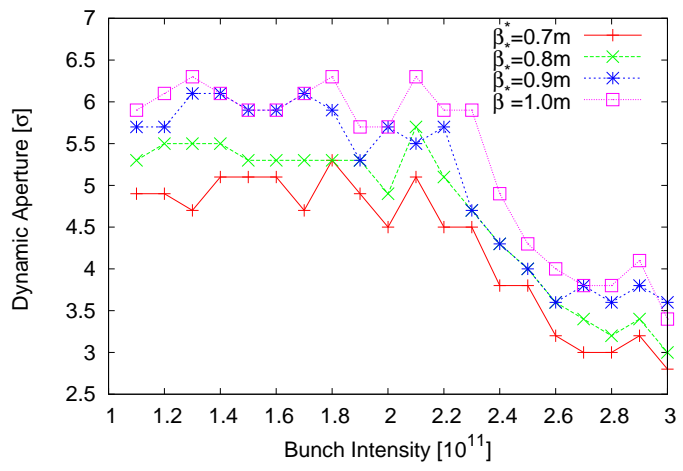

Figure 24: Calculated dynamic apertures versus the proton bunch intensity for different $\beta^{*}$ s. Particle energy is $100 \mathrm{GeV}$.

With beam-beam interaction, by pushing the tunes close to the third order resonances, we could reduce the beam loss by adjusting the local IR sextupole correctors. However, with beam-beam interaction on, the effect of these corrections are not conclusive. Possible explanation is that the beam lifetime with collisions is mainly determined by beam-beam interaction, even though the third order resonance correction could possibly yield some tune space.

\subsection{Limited Tune Space}

In the $2013255 \mathrm{GeV}$ p-p run, to gain more tune space for ever increased bunch intensity, we moved the lattice tunes up towards $7 / 10$. However, the store polarization measurements showed a significant reduction when the vertical tune was above 0.686 . Larger beam losses were observed from time to time when the tunes were too high. To maximize the FOM in the proton operation, we had to cut down the bunch intensity to below $1.85 \times 10^{11}$ and place the vertical tune below 0.686 for the rest 2013 run.

Currently we are upgrading the polarized proton source [67]. Once it is complete, the bunch intensity is expected to reach $3 \times 10^{11}$ in RHIC. Then the total beam-beam parameter will be 0.03 . According to Fig. 4 , there will be not enough tune space between $2 / 3$ and $7 / 10$ to accommodate such a big beam-beam tune spread.

Figure 24 shows the calculated dynamic aperture versus the proton bunch intensity. In this simulation, to make a full usage of the available tune space between $2 / 3$ and $7 / 10$, we place the zero-amplitude particle tunes with beam-beam interaction to $(0.675,0.67)$. The particle energy is $100 \mathrm{GeV}$. The normalized rms transverse emittance is $2.5 \mu \mathrm{m}$. The initial relative off-momentum deviation is $12.5 \times 10^{-4}$. From the plot, the dynamic aperture begins to drop for all $\beta^{*} \mathrm{~s}$ when the bunch intensity is larger than $2 \times 10^{11}$, or the total beam-beam parameter is larger than 0.02 .

Massive numerical simulations and detailed beam experiments had been carried out to search for a new tune space which provides a good beam lifetime with a large beam-beam parameter and a good proton polarization transmission efficiency on the ramp as well as a good polarization lifetime at store. For an example, in the $2008100 \mathrm{GeV}$ p-p run, we tested a near-integer working point $(0.96,0.95)$ in the Blue ring [7]. We observed a huge background in the detector due to a $10 \mathrm{~Hz}$ horizontal orbit oscillation. Since it was not possible to overcome the $10 \mathrm{~Hz}$ orbit oscillation in a short time, we abandoned the near-integer working point in that run.

\subsection{Head-on Beam-beam Compensation}

A possible solution to reduce the beam-beam tune spread is head-on beam-beam compensation [68, 69, 70, 71]. The idea is to introduce a low energy electron beam to collide head-on with the proton bunches. The electron beam has a same rms transverse beam size as the proton bunches. The device to provide such an electron beam is called an electron lens (e-lens). A pulsed e-lens had been installed and operated in the Tevatron to compensate the long-range beam-beam tune shifts, and to clean the de-bunched beam and halo particles $[72,73,74,75,76]$.

In our design [70], a DC electron beam is adopted. Two e-lenses are needed, one for the Blue ring and one for the Yellow ring. They are installed on either side of IP10 where the two proton beams are separated 


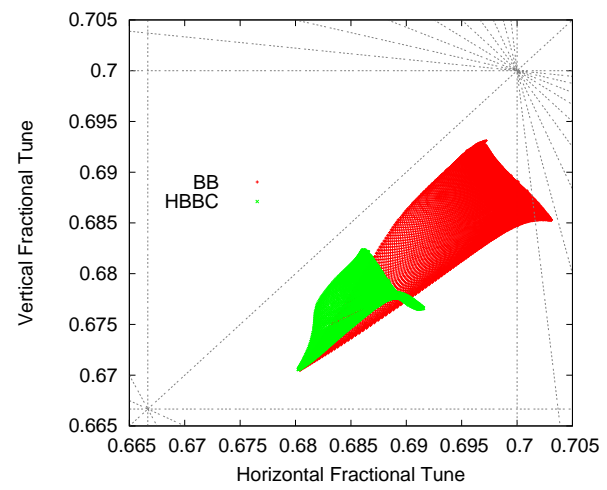

Figure 25: Tune footprints without beam-beam compensation and with half beam-beam compensation. The bunch intensity is $2.5 \times 10^{11}$.

vertically. To stabilize the electron beam, the compensation region is surrounded by a superconducting solenoid with a magnetic field up to $6 \mathrm{~T}$. To cancel their effects on the global betatron coupling and spin dynamics, the two solenoids are powered with opposite polarities.

Figure 25 shows the tune footprints without compensation and with half beam-beam compensation with a bunch intensity $2.5 \times 10^{11}$. Half and full compensation compensate half and full total beam-beam tune spread respectively. Simulations show that half beam-beam compensation gives a larger dynamic aperture than full head-on beam-beam compensation [71]. The reason is that full compensation introduces additional nonlinearities into the ring. To cancel the nonlinear beam-beam resonance RDTs from the proton-proton interaction at IP8, we set the phase advances between IP8 and the center of e-lenses to be $k \pi, k$ is an integer.

From the previous RHIC p-p operation, the beam lifetime is mainly determined by the limited transverse off-momentum dynamic aperture which is reduced by beam-beam interaction. Therefore, the candidate lattices for head-on beam-beam compensation should have small nonlinear chromaticities and a large offmomentum dynamic aperture. The ATS scheme for the HL-LHC is tested and chosen for the RHIC p-p operation with beam-beam compensation.

\section{Other Beam-beam Observations}

\section{1 $10 \mathrm{~Hz}$ Orbit Oscillation}

In the $2008100 \mathrm{GeV}$ p-p run, we tested a near-integer working point $(0.96,0.95)$ in the Blue ring while keeping the working point in the Yellow ring at $(0.695,0.685)$. Near the integer resonance, it was difficult to control the closed orbit, $\beta$-beat, tunes, and betatron coupling. With collisions at store, a large background and a large oscillation in the experiment rates were observed, which was found to be caused by a $10 \mathrm{~Hz}$ orbit oscillation.

The horizontal beam orbit oscillation at frequencies around $10 \mathrm{~Hz}$ has been observed at RHIC for several years. It had been amplified with the near integer tune. The distinct frequencies of this jitter have been found to originate from the superconducting quadrupole triplet magnets around the ring, and coincide with mechanical vibration modes of the cold masses. We identified the liquid helium flow as the driving force of these oscillations. The amplitude of the $10 \mathrm{~Hz}$ orbit oscillation is approximately one tenth of the rms beam size. $10 \mathrm{~Hz}$ orbit oscillation also causes a $10 \mathrm{~Hz}$ tune oscillation. The observed maximum peak-to-peak tune modulation could reach $1.0 \times 10^{-3}$ on the energy ramp.

To correct the $10 \mathrm{~Hz}$ orbit oscillations, we developed a fast $10 \mathrm{~Hz}$ orbit correction feedback system with 12 local horizontal dipole correctors in the IRs. This system became operational in the 2011 p-p run [18]. The top and bottom plots of Fig. 26 shows the Zero-Degree-Calorimeter (ZDC) coincidence rates from the Phenix detector and the horizontal BPM readings from bo-bh1. BPM bo-bh1 is the closest one to IP6 in the Blue ring. The peak-to-peak amplitude of $10 \mathrm{~Hz}$ oscillations were $1200 \mathrm{um}$ without correction. With $10 \mathrm{~Hz}$ orbit feedback on, it reduced to $80 \mathrm{um}$. The $10 \mathrm{~Hz}$ orbit feedback reduced the detector noise level in the ZDC rates too. 

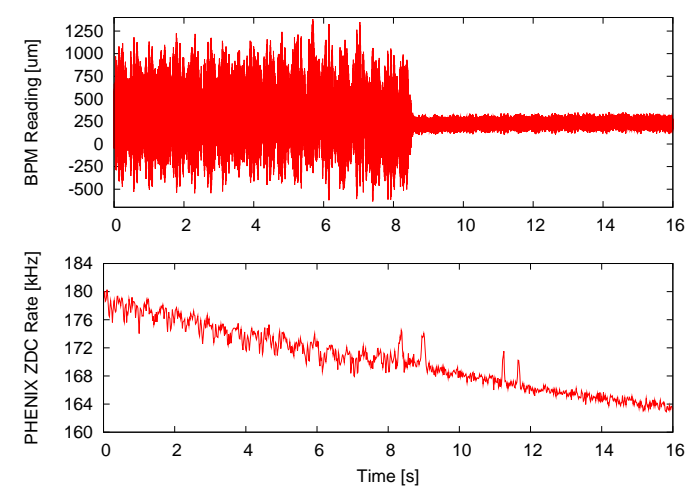

Figure 26: Phenix ZDC rates (top) and BPM readings (bottom) without and with $10 \mathrm{~Hz}$ orbit feedback on.

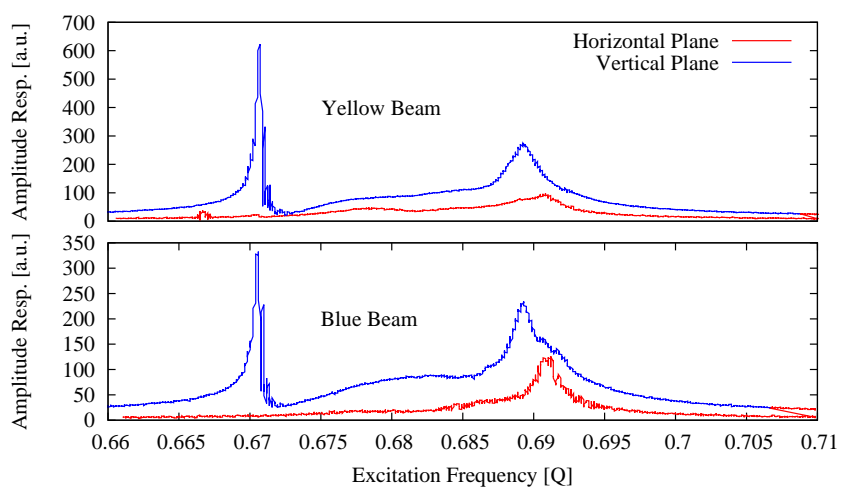

Figure 27: An example of beam-beam BTF measurements for the Yellow ring (Upper) and for the Blue ring (bottom).

\subsection{Coherent Beam-beam Modes}

The first successful measurement of coherent beam-beam modes in RHIC took place in the $23 \mathrm{GeV} \mathrm{Au}-\mathrm{Au}$ run in 2002 [77]. In the routine RHIC operation, a base-band PLL tune meter is used to measure the coherent beam-beam modes. By scanning the kicking frequency of the PLL tune meter, we record the beam transfer function (BTF). The beam-beam BTF is measured every 30 minutes during physics stores. One application of beam-beam BTF is to estimate the total beam-beam parameter [78].

Figure 27 shows an example of BTF measurements acquired during a store with 12 bunches colliding at IP6 and IP8 in the $2012100 \mathrm{GeV}$ p-p run. The top plot is the beam-beam BTFs taken in the Yellow ring, and the bottom one in the Blue ring. In each ring, both horizontal and vertical BTFs were shown. In Fig. 27 , the horizontal axis is the PLL kicking frequency in units of revolution frequency. For each spectrum, the peak with a higher frequency shows the $\sigma$-mode, while the lower frequency peak shows the the $\pi$-mode. In Fig. 27, the $\pi$-mode was missing in the horizontal plane, which could be explained by a transfer of Landau damping from one plane to the other.

Dedicated beam experiments were carried out in the 2012 p-p runs to study the effects of coherent beam-beam modes and possible suppression techniques [79]. Since the coherent beam-beam mode is located at about 1.3 times beam-beam parameter from the unperturbed tunes for two identical round Gaussian bunches, we could expect that it will further reduce the limited tune space between $2 / 3$ and $7 / 10$. In one experiment, we deliberately moved the tunes of the Blue ring toward $Q_{y}=2 / 3$ resonance while keeping the tunes in the Yellow ring untouched. A large beam loss was only seen in the Blue ring when the $\pi$-mode was located at 0.669. There was no clear transverse emittance blow-up. Since the beam loss only happened in one ring, the beam loss observed in the Blue ring is not caused by coherence beam-beam mode [79]. In the routine physics run in 2013, the $\pi$ mode could even reach 0.667 right after collision. 


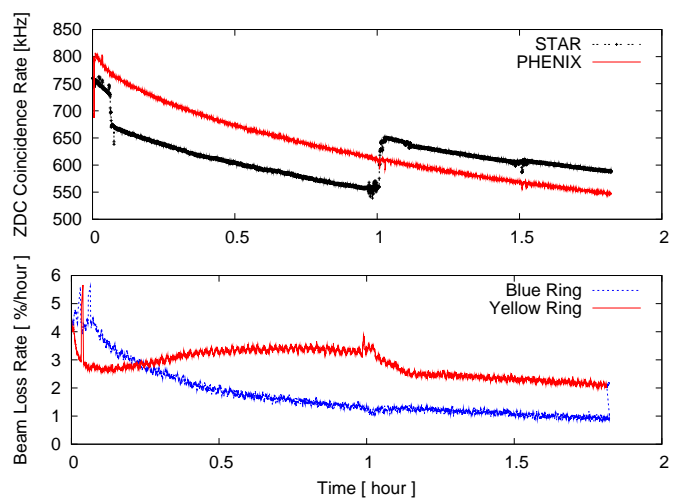

Figure 28: ZDC rates (upper) and beam loss rates (bottom) during offset collision in the $2013255 \mathrm{GeV}$ p-p run. The beam in the Blue ring was offset horizontally by $90 \mu \mathrm{m}$, or $1 \sigma$ during the first hour of store.

\subsection{Offset Collision}

Dedicated beam experiments to study the emittance growth with offset collisions in RHIC took place during the $2004 \mathrm{Au}-\mathrm{Au}$ run and p-p run [80]. The experiments were performed at the end of stores when the luminosity decay can be well fitted and therefore extrapolated. The beams were offset at the collisional points for $15 \mathrm{~min}$. By comparing the extrapolated luminosity after the offset was removed, the change in emittance growth can be deduced. For a small beam-beam parameter of 0.003 , we did not detect any additional transverse emittance growth with up to $1.1 \sigma$ transverse offset.

During the 2013 run, luminosity leveling with offset collision was adopted at the beginning of physics store. The beam-beam parameter was 0.014 . We only offset the beam in the Blue ring transversely at IP6 by $90 \mu \mathrm{m}$, or $1 \sigma$. Figure. 28 shows the Star ZDC rates and the particle loss rates in both rings. The ZDC rate from Star dropped by $10 \%$ with offset collision, which agreed with its analytical prediction. With offset collision, there was slightly more particle loss in the Yellow ring. While in the Blue ring, the offset did not cause additional visible particle loss. Compared to the stores wuth and without offset collisions, we did not observe conclusive emittance growth due to offset collisions.

\section{CONCLUSIONS}

In this article, we presented the beam-beam observations at routine physics stores in the previous RHIC p-p operation. The particle loss took place in the transverse plane instead of leaking out of RF buckets. We found a strong linear correlation between the beam loss rate and the particle leakage rate from the central bunch area. We concluded that the particle loss in the routine physics stores was due to a limited off-momentum dynamic aperture which was reduced by beam-beam interaction. Simulation of dynamic aperture with beam-beam interaction confirmed this.

We analyzed the mechanisms for the growths of transverse emittances and bunch lengths at store. IBS effect is the main source to move particles out of the central bunch area, especially at the beginning of store when the particle line density at the bunch center was high. Numeric simulations with IBS can largely reproduce the evolutions of transverse emittances and bunch lengths.

Current working point is constrained between $2 / 3$ and $7 / 10$ in the tune space. Before reaching the beam-beam tune space limit, the transverse dynamic aperture with beam-beam interaction in the RHIC p-p operation was reduced by the local IR multipole field errors, chromatic effects with low $\beta^{*}$ lattices, and third order resonances at $Q_{x, y}=2 / 3$.

With increased bunch intensities in the 2013 p-p run, we used up all possible tune space between $2 / 3$ and $7 / 10$. Transverse emittance blow-ups were regularly observed right after collision. To maximize the FOM of p-p operation, we had to limit the maximum proton bunch to $1.85 \times 10^{11}$ in the routine operation. Head-on beam-beam compensation was proposed and two electron lenses are being installed in RHIC to reduce the beam-beam tune spread. 


\section{ACKNOWLEDGEMENTS}

The authors would like to thank our colleagues M. Bai, M. Blaskiewicz, H. Huang, M. Minty, C. Montag, V. Ptitsyn, V. Ranjbar, V. Scheoffer, and T. Roser for stimulating discussions during the RHIC polarizaed proton operation. The authors also would like to thank V. Lebedev, V. Shiltsev, A. Valishev, and many others for discussions. This work was supported by the US DOE under contract No. DE-AC02-98CH10886.

\section{References}

[1] W. Fischer, RHIC Run Overview, website: http://www.rhichome.bnl.gov/RHIC/Runs/.

[2] T. Roser, et al., Workshop on Increasing the AGS Polarization, University of Michigan (2002).

[3] H. Huang, et al., Proceedings of the 2003 Particle Accelerator Conference, Portland, OR, USA (2003).

[4] M. Bai, et al., Proceedings of 16th International Spin Physics Symposium, Trieste, Italy (2004).

[5] M. Bai, et al., Proceedings of the 2005 Particle Accelerator Conference, Knoxville, TN, USA (2005).

[6] V. Ptitsyn, et al., Proceedings of the 2006 European Particle Accelerator Conference, Edinburgh, UK (2006)

[7] C. Montag, et al., Proceedings of the 2008 European Particle Accelerator Conference, Genoa, Italy (2008).

[8] C. Montag, et al., Proceedings of the 2010 International Particle Accelerator Conference, Kyoto, Japan (2010).

[9] V. Schoefer, et al., Proceedings of the International Particle Accelerator Conference 2012, New Orleans, Louisiana, USA (2012).

[10] M. Bai, et al., Phys. Rev. Lett. 96, 174801 (2006).

[11] M. Bai, et al., Proceedings of the 2009 Particle Accelerator Conference, Vacouver, BC, Canada (2009).

[12] H. Huang, et al., Proceedings International Particle Accelerator Conference 2011, San Sebastian, Spain, pp. 1888-1890 (2011).

[13] H. Huang, et al., Proceedings of the 2011 Particle Accelerator Conference, NY, New York, USA, pp. 41-45 (2011).

[14] V. Schoefer, et al., Proceedings of the International Particle Accelerator Conference 2012, New Orleans, Louisiana, USA, pp. 184-186 (2012).

[15] V.H. Ranjbar, et al., Proceedigns of the International Particle Accelerator Conference 2013, Shanghai, China (2013).

[16] Y. Luo, et al., Phys. Rev. ST Accel. Beams 9, 124001 (2006) Published 14 December 2006

[17] P. Cameron, et al., Phys. Rev. ST Accel. Beams 9, 122801 (2006) Published 14 December 2006

[18] R. Michnoff, et al., Proceedings of the 2011 Particle Accelerator Conference, New York City, NY, USA, pp. 41-45 (2011).

[19] M. Michiko, et al., Proceedings of the 2011 Particle Accelerator Conference, New York, NY, March 28April 1, 2011,

[20] W. Fischer, et al., Phys. Rev. ST Accel. Beams 5, 124401, 2002 .

[21] W. Fischer, et al., Phys. Rev. ST Accel. Beams 11, 041002, 2008.

[22] S. Y. Zhang, et al., Phys. Rev. ST Accel. Beams 8, 123201, 2005.

[23] S. Y. Zhang and V. Ptitsyn, Phys. Rev. ST Accel. Beams 11, 051001, 2008. 
[24] H. Kim, et al., Phys. Rev. ST Accel. Beams 12, 031001 (2009).

[25] R. Calaga, et al., Phys. Rev. ST Accel. Beams 14, 091001 (2011).

[26] K. Hirata, edited by A. Chao, K. H. Mess, M. Tigner, and F. Zimmermann (World Scientific, Singapore, 2013).

[27] T. Sen, et al., Phys. Rev. ST Accel. Beams 7, 041001 (2004).

[28] W. Chao, Lecture Notes on Topics in Accelerator Physics, March 2001 (unpublished).

[29] B.V. Chirikov, Physics Report 52, No 5 (1979).

[30] L.R. Evans and J. Gareyte, CERN Yellow Report 87-03, 1987.

[31] J. Laskar, in Proceedings of the 2003 Particle Accelerator Conference, Portland, Oregon.

[32] Y. Papaphilippou and F. Zimmermann, Phys. Rev. ST Accel. Beams 2, 104001 (1999).

[33] Y. Papaphilippou and F. Zimmermann, Phys. Rev. ST Accel. Beams 5, 074001 (2002).

[34] V. Shiltsev, et al., Phys. Rev. ST Accel. Beams 8, 101001 (2005).

[35] A. Wolski, edited by A. Chao, K. H. Mess, M. Tigner, and F. Zimmermann (World Scientific, Singapore, 2013).

[36] Y. Luo, Proceedings of the 2010 International Particle Accelerator Conference, Kyoto, Japan (2010).

[37] Y. Luo, Proceedings of the 2015 International Particle Accelerator Conference, Richmond, VA, USA (2015).

[38] M. J. Rhoades-Brown and M. Harrison, BNL Report No. 47070, 1991 (unpublished).

[39] D. Trbojevic, BNL RHIC AP Report No. 136, 1997 (unpublished).

[40] L. D. Landau and E. M. Lifshitz, Mechanics (Pergamon Press, Oxford, 1960).

[41] J. D. Jackson, Classic Electrodynamics (John Wiley- Sons, New York, 1975).

[42] B. Frankzke, CERN Yellow Report No. 92-01, 1992, pp. 100120.

[43] V. Lebedev, in Handbook of Accelerator Physics and Engineering, edited by A. Chao, K. H. Mess, M. Tigner, and F. Zimmermann (World Scientific, Singapore, 2013).

[44] C.Bernardini, et al., Phys. Rev. Lett. 10., 407 (1963).

[45] J. Haissinski, Rapport technique interne No. 41-63, LAL ORSAY (1963).

[46] J. Le Duff, Proceeding of the CERN Accelerator School, Berlin, 1987.

[47] A. Piwinski, DESY 98-179, 1998.

[48] A. Piwinski, in Frontiers of Particle Beams, edited by M. Month and S. Turner (Springer, New York, 1988), p. 297.

[49] J. Bjorken and S. Mtingwa, Part. Accel. 13, 115 (1983).

[50] M. Martini, Part. Accel. 17, 1 (1985).

[51] Y. Luo, et al., Phys. Rev. ST Accel. Beams 17, 081003 (2014).

[52] A. Fedotov, BNL RHIC/AP/168, 2009.

[53] R. Tomas, et al., in the 2004 Proceedings of European Particle Accelerator Conference, Lucerne, Switzerland (2004).

[54] J. Beebe-Wang and A. Jain, in Proceedings of PAC 2007, Albuquerque, New Mexico, USA. 
[55] J-P. Koutchouk, et al., Proceedings of the 2001 Particle Accelerator Conference, Chicago, USA (2001).

[56] Fulivia, Proceedings of the 2009 Particle Accelerator Conference, Vacouver, BC, Canada (2009).

[57] J. Wei, Particle Accelerators, Vol.55, pp. 439-448, 1996.

[58] W. Fischer, et al., BNL C-A/AP/471, 2012 (unpublished).

[59] Y. Luo, et al., BNL C-A/AP/419, 2011 (unpublished).

[60] Y. Luo, Proceedings of the 2011 Particle Accelerator Conference, New York, NY, 2011, p.2235-2237.

[61] Y. Luo, Proceedings of the 2009 Particle Accelerator Conference, Vacouver, BC, Canada (2009).

[62] Y. Luo, Proceedings of the 2011 Particle Accelerator Conference, New York, NY, 2011, p.2232-2234,

[63] S. Fartoukh, Phys. Rev. ST Accel. Beams 16, 111002, 2013.

[64] R. Tomas, et al., Phys. Rev. ST Accel. Beams 8, 024001

[65] Y. Luo, et al., Proceedings of the 2007 Particle Accelerator Conference, Albuquerque, New Mexico (2007).

[66] Y. Luo, et al., BNL C-A/AP/264, 2006 (unpublished).

[67] A. Zelenski, Rev. Sci. Instrum. 81, $02 B 308$ (2010).

[68] W. Fischer, in the 2005 RHIC Accelerator Physics Experiments (APEX) Workshop, 2005, Upton, NY.

[69] V. Shiltsev, in the 2005 RHIC APEX workshop, 2005, Upton, NY.

[70] Y. Luo and W. Fischer, BNL C-A/AP/286, 2007 (unpublished).

[71] Y. Luo, Phys. Rev. ST Accel. Beams 15, 051004 (2012).

[72] V. Shiltsev, et al., Phys. Rev. ST Accel. Beams 2, 071001 (1999).

[73] V. Shiltsev, et al., Phys. Rev. Lett. 99, 244801 (2007).

[74] V. Shiltsev, et al., Phys. Rev. ST Accel. Beams 11, 103501 (2008).

[75] X. Zhang, et al., Phys. Rev. ST Accel. Beams 11, 051002 (2008).

[76] G. Stancari, Proceeding of the 2013 North American Particle Accelerator Conference, Pasadena, California,USA (2013).

[77] W. Fischer, et al., Proceedings of the 2003 Particle Accelerator Conference, Portland, OR, USA (2003).

[78] K. Yokoya, et al., Particle Accelerators, Vol.27, p.181, 1990.

[79] S. White, Proceeding of the 2012 International Particle Accelerator Conference, New Orleans, Louisiana, USA (2012).

[80] N. Abreu, et al., BNL C-A/AP/284, 2007 (unpublished).. 